\title{
Tracking multi-directional intersecting cracks in numerical modelling of masonry shear walls under cyclic loading
}

\author{
Savvas Saloustros · Miguel Cervera · Luca Pelà
}

\begin{abstract}
In-plane cyclic loading of masonry walls induces a complex failure pattern composed of multiple diagonal shear cracks, as well as flexural cracks. The realistic modelling of such induced localized cracking necessitates the use of costly direct numerical simulations with detailed information on both the properties and geometry of masonry components. On the contrary, computationally efficient macro-models using standard smeared-crack approaches often result in a poor representation of fracture in the simulated material, not properly localized and biased by the finite element mesh orientation. This work proposes a possible remedy to these drawbacks of macro-models through the use of a crack-tracking algorithm. The macro-modelling approach results in an affordable computational cost, while the tracking algorithm aids the mesh-bias independent and localized representation of cracking. A novel methodology is presented that allows the simulation of intersecting and multi-directional cracks using tracking algorithms. This development extends the use of localized crack approaches using tracking algorithms to a wider field of applications exhibiting multiple, arbitrary and interacting cracking. The paper presents
\end{abstract}

Savvas Saloustros · Miguel Cervera · Luca Pelà

Department of Civil and Environmental Engineering Universitat Politècnica de Catalunya (UPC-BarcelonaTech) Jordi Girona 1-3, 08034 Barcelona, Spain.

International Center for Numerical Methods in Engineering (CIMNE)

Gran Capità, S/N, 08034 Barcelona, Spain.

E-mail: savvas.saloustros@upc.edu

E-mail: miguel.cervera@upc.edu

E-mail: luca.pela@upc.edu also a novel formulation including into an orthotropic damage model the description of irreversible deformations under shear loading. The proposed approach is calibrated through the comparison with an experimental test on a masonry shear wall against in-plane cyclic loading.

Keywords Continuum Damage Mechanics · CrackTracking · Cyclic Shear Loading · Intersecting Cracks · Masonry walls

\section{Introduction}

Damage in masonry appears in the form of localized cracks. The propagation of these cracks within the structure determines the development of the collapse mechanism and consequently the capacity of the structure under the given loading conditions. This structural behaviour has been taken into account for the development of both classical and advanced analysis tools for the structural assessment of masonry constructions $[1,2,3,4]$.

Numerical modelling of masonry structures has undergone important developments in the last decades. The inventory of numerical methods today includes simplified techniques such as limit analysis software $[5,6,7,8]$, equivalent frame methods $[9,10,11]$ and macro-modelling approaches $[12,13,14]$ to sophisticated ones as micro-modelling $[15,16]$ and multi-scale finite element models [17, 18, 19]. Simplified numerical methods, like the macro-modelling ones, consider masonry as a continuum material with average properties. This simplification allows for the numerical analysis of large scale structures at an affordable computational cost with however limited accuracy in the damage representation. Reciprocally, the explicit consideration of the masonry micro-structure in micro-modelling 
techniques results in a more realistic representation of cracking, at the cost of increased computation time and memory requirements that limits up to date their application to small structural elements rather than whole structures.

An affordable alternative to the simulation of localized cracking in large scale structures was proposed by Cervera and co-workers [20, 21, 22]. The method bases on the enhancement of the smeared crack approach with the use of a crack-tracking algorithm. The outcome of this approach is the realistic representation of evolving localized cracks within the analysed structure for a computational cost comparable to that of the classical smeared crack approach [23, 24].

Despite the above recent applications on masonry structures, tracking algorithms have a long history in the simulation of localized failure in quasi-brittle materials. They are used for the enhancement of problems involving the progression of both weak, or smeared (e.g. $[25,26,22])$ and strong, or discrete, discontinuities (e.g. $[27,28,29,30])$. Independently of the chosen approach, their application aims to circumvent the problem of the mesh-dependency that the irreducible standard finite

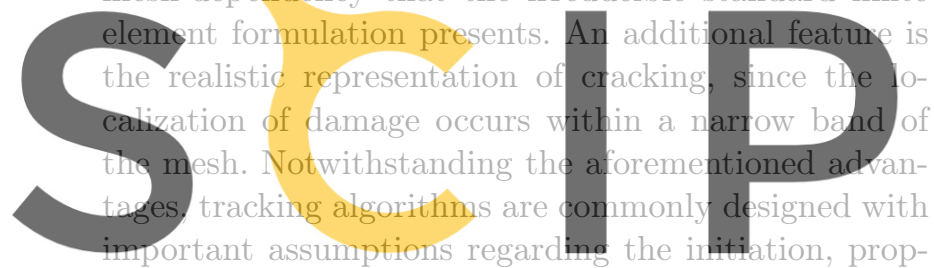
agation and completion of the simulated cracks. Such

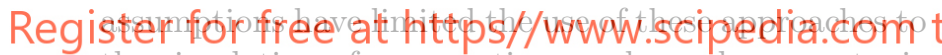
the simulation of propagating cracks under monotonic loading.

This paper presents a methodology to simulate efficiently the localized cracking in masonry structures induced by in-plane cyclic loading using a continuum damage mechanics model enhanced with a cracktracking algorithm. Cyclic loading of masonry structures may result in complex damage patterns characterized by multiple and intersecting cracks. To appropriately model this, a novel numerical methodology is proposed, on the basis of the tracking algorithm developed in previous works $[22,31,32]$, that allows the simulation of intersecting and multi-directional cracking, extending the use of tracking algorithms to a large field of applications. At the constitutive level, the paper presents a novel formulation in the context of a continuum damage model for the description of irreversible deformations under shear loading.

After this introduction, the paper is organized in the following way. Section 2 presents the used constitutive damage model. Following that, the novel tracking algorithm is detailed in Section 3. The proposed approach is calibrated in Section 4 through the simulation of an experimentally tested masonry wall under cyclic shear loading. The same section addresses the issue of mesh objectivity of the tracking algorithm. The paper ends with the concluding Section 5 .

\section{Constitutive model}

The material non-linearity is represented in the numerical simulations through the use of the constitutive model presented in [33]. It is a strain-based continuum damage model with two scalar damage variables for distinguishing between tensile and compressive damage. The decomposition of the effective stress tensor into positive and negative parts, as well as the use of the two damage variables, results in a unilateral effect under tension-compression cyclic loading. The model can account for irreversible deformations through a simple and efficient algorithm, within the context of continuum damage mechanics. The above properties, as well as the limited number of involved material parameters and the simple explicit formulation, make it a suitable model for the seismic analysis of large concrete and ma-
sonry structures $[34,35,36,37]$.
For the sake of completeness, this section presents
the basic ingredients of the aforementioned tension-
compression damage model. The shear behaviour of the
moctel is cerefully considered through the proper defi-
nition of the damage surfaces and the evolution of the irreversible strains. The modelling of the irreversible downiload the byensioniw ithouts thenwatersmark mainly intended for concrete structures in [33, 34, 38]. The section ends with a discussion over the effect of the proposed alterations to the evolution of the irreversible deformations under shear loading.

\subsection{Constitutive law}

The Cauchy stress tensor (of rank two) $\boldsymbol{\sigma}$ is computed in the tension-compression damage model through the constitutive relation

$$
\boldsymbol{\sigma}=\left(1-d^{+}\right) \overline{\boldsymbol{\sigma}}^{+}+\left(1-d^{-}\right) \overline{\boldsymbol{\sigma}}^{-}
$$

where $d^{+}$and $d^{-}$are scalar variables denoting the compressive and tensile damage affecting the positive $\overline{\boldsymbol{\sigma}}^{+}$ and negative parts $\overline{\boldsymbol{\sigma}}^{-}$of the effective stress tensor respectively. The latter are calculated through the distinguishing stress split according to [38]

$$
\overline{\boldsymbol{\sigma}}^{+}=\sum_{j=1}^{3}\left\langle\bar{\sigma}_{j}\right\rangle \mathbf{p}_{\mathbf{j}} \otimes \mathbf{p}_{\mathbf{j}}
$$


$\overline{\boldsymbol{\sigma}}^{-}=\overline{\boldsymbol{\sigma}}-\overline{\boldsymbol{\sigma}}^{+}$

In the above equations, $\sigma_{j}$ is the principal stress corresponding to the eigenvector $\mathbf{p}_{\mathbf{j}}$ of the effective stress tensor $\overline{\boldsymbol{\sigma}}$ and the symbols $\langle\cdot\rangle$ are the Macaulay brackets $(\langle x\rangle=x$, if $x \geq 0,\langle x\rangle=0$, if $x<0)$.

The model uses two scalar variables $\tau^{ \pm}$, the equivalent stresses, to distinguish between loading, unloading and reloading. The adopted values of $\tau^{+}$and $\tau^{-}$determine the shape of the $d^{+}$and $d^{-}$damage surfaces. In this work we have adopted the values proposed in $[19,39]$

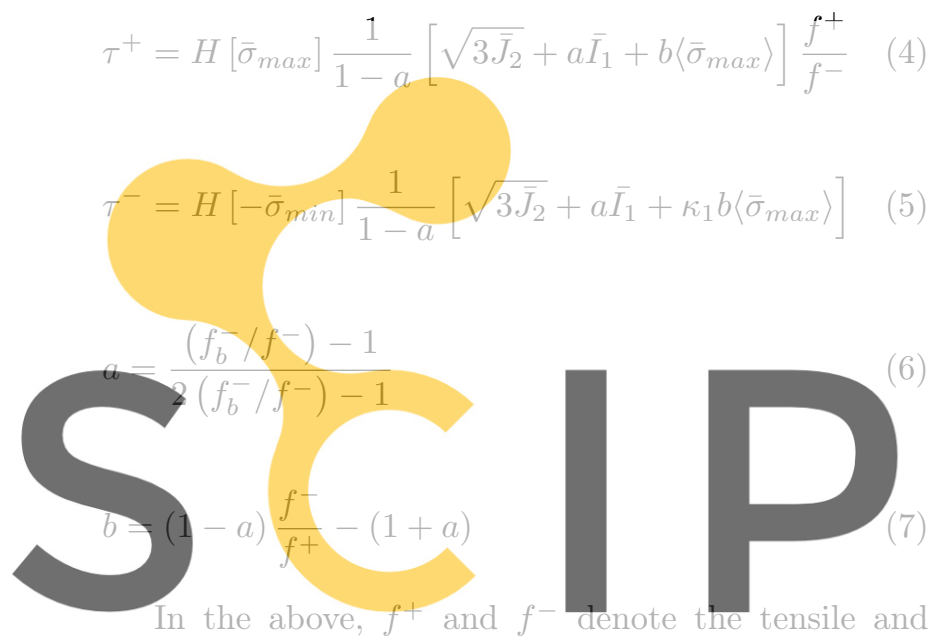
compressive uniaxial strengths respectively and $f_{b}^{-}$the

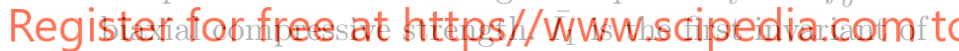
the effective stress tensor and $\bar{J}_{2}$ the second invariant of the deviatoric effective stress tensor. Finally, $\bar{\sigma}_{\max }$ and $\bar{\sigma}_{m i n}$ stand for the maximum and minimum principal effective stresses respectively. The use of the Heaviside step function $(H[x]=1$ if $x \geq 0$ and $H[x]=0$ if $x<0)$ activates the $\tau^{+}$for tensile and shear stress states (1st, 2nd and 4th quadrants in Figure 1), and the $\tau^{-}$for compressive and shear shear stress states (2nd, 3rd and 4th quadrants in Figure 1).

The $\kappa_{1}$ variable controls the shape of the failure surface in the shear quadrants (i.e. 2nd and 4th quadrants in Figure 1). Its value varies between 0 (i.e. the Drucker-Prager criterion) and 1 (i.e. the criterion proposed in [40]). Note that the use of the Macaulay brackets for $\bar{\sigma}_{\max }$ in equation (5) makes the function $\tau^{-}$independent of $\kappa_{1}$ for pure compression. This, in addition to the fact that $\kappa_{1}$ does not appear in equation (4), make the tensile and compressive response in the 1st and 3rd quadrants of Figure 1 independent of $\kappa_{1}$. As shown by Petracca et al. [39, 41], the different choice in the definition of the equivalent stresses from those proposed in $[35,38,40]$ results in a better control of the model behaviour under shear monotonic loading. As it will be shown later, the choice of such surfaces in the current model results also in the consideration of the irreversible deformations under shear loading.
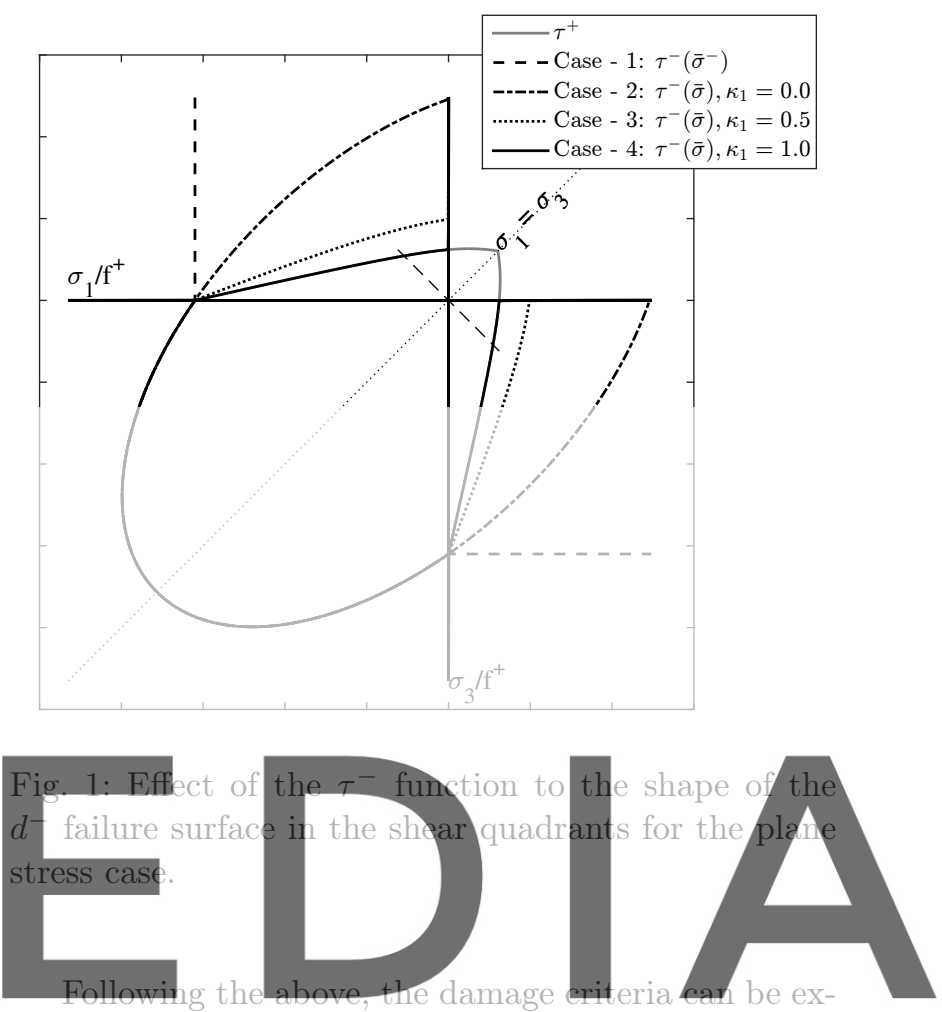

pressed as

\section{download the version without the watermark}

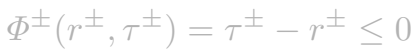

where the internal variables $r^{+}$and $r^{-}$represent the current damage thresholds and the respective expansion of the $d^{+}$and $d^{-}$damage surfaces. Their initial values are defined to be equal to the uniaxial tensile strength $r_{0}^{+}=f^{+}$and the uniaxial compressive strength $r_{0}^{-}=$ $f^{-}$. After damage is triggered, their values are equal to the maximum attained values by the equivalent stresses

$r_{n}^{ \pm}=\max \left[r_{0}^{ \pm}, \max _{i \in(0, n)}\left(\tau_{i}^{ \pm}\right)\right]$

with $n$ representing the number of the current (load /displacement) increment.

The effect of the irreversible deformations is accounted using the following definition of the effective stress tensor $\overline{\boldsymbol{\sigma}}$

$\overline{\boldsymbol{\sigma}}=C_{\mathbf{0}}:\left(\boldsymbol{\epsilon}-\boldsymbol{\epsilon}^{i}\right)=C_{\mathbf{0}}: \boldsymbol{\epsilon}^{e}$ 
where $\boldsymbol{C}_{\mathbf{0}}$ denotes the (rank four) elastic constitutive tensor and $\epsilon^{i}$ the irreversible part of the second order total strain tensor $\boldsymbol{\epsilon}$ and $\boldsymbol{\epsilon}^{e}$ the elastic one. Contrary to the total strain tensor, which is an external variable of the model, the irreversible strain tensor is an internal variable. The following expression is proposed in this work for the rate of the irreversible strains

$\dot{\boldsymbol{\epsilon}}^{i}=\lim _{\Delta t \rightarrow \infty}\left(\frac{\beta}{\Delta t} \frac{r^{-}(\tilde{\tilde{\boldsymbol{\sigma}}})-r_{t}^{-}}{r_{t+\Delta t}^{-}} \boldsymbol{\epsilon}_{t+\Delta t}^{e}\right)$

or in incremental form as

$$
\Delta \epsilon^{i}=\beta \frac{r^{-}(\tilde{\overline{\boldsymbol{\sigma}}})-r_{n}^{-}}{r_{n+1}^{-}} \epsilon_{n+1}^{e}
$$

In the above equations, $n$ and $n+1$ subscripts refer to subsequent time steps. The symbol " $\Delta$ " denotes the increment of a specific quantity from step $n$ to $n+1$. The variables $\tilde{\bar{\sigma}}$ and $r^{-}(\tilde{\bar{\sigma}})$ represent, respectively, the effective stress and the stress threshold in the absence of increment of irreversible strains at time step $n+1$

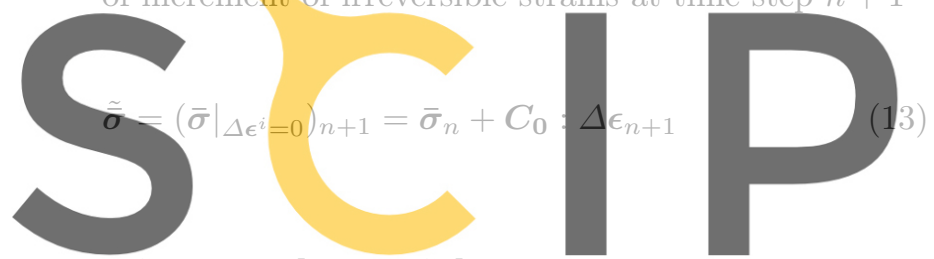

$r(\tilde{\bar{\sigma}})=\max \left[r_{n}^{-}, \tau^{-}(\tilde{\bar{\sigma}})\right]$

(14) $\lambda=1-\beta\left(1-\frac{r_{n}^{-}}{r^{-}(\tilde{\overline{\boldsymbol{\sigma}}})}\right)$

The computation of the effective stresses at each increment of the analysis is presented in the flowchart of Table 1.

(i) Compute $\tilde{\overline{\boldsymbol{\sigma}}}$ using equation (13).

(ii) Is $\beta>0$ ?

YES: Continue to step (iii)

NO: No evolution of irreversible strains is considered. Set $\overline{\boldsymbol{\sigma}}_{n+1}=\tilde{\overline{\boldsymbol{\sigma}}}$ and EXIT.

(iii) Calculate $\tau^{-}(\tilde{\overline{\boldsymbol{\sigma}}})$ using equation (5) and $r^{-}(\tilde{\tilde{\boldsymbol{\sigma}}})$ using equation (14).

(iv) Compute $\lambda$ using equation (17). Set $\bar{\sigma}_{n+1}=\lambda \tilde{\bar{\sigma}}$ and EXIT

Table 1: Flowchart for the calculation of the effective stresses.
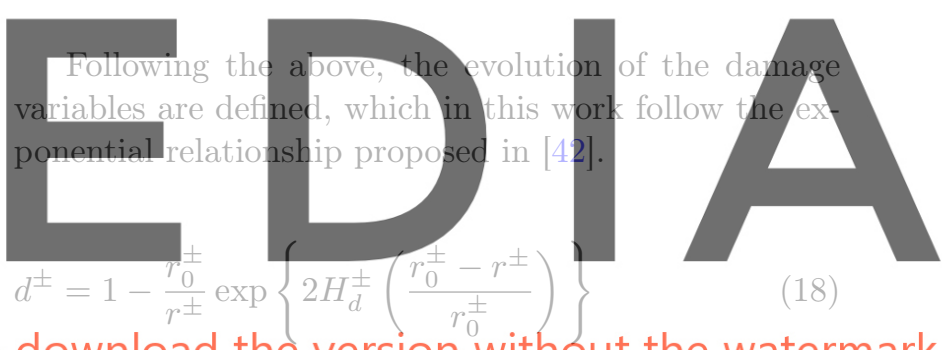

(18)

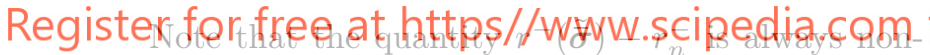
negative due to equation (14), an essential thermodynamic requirement for ensuring non-negative dissipation. Finally, the material parameter $\beta$, with values between 0 and 1 , defines the rate of the irreversible deformation by scaling the current increment of the equivalent stresses, and consequently the stress thresholds, through the following equation

$$
\begin{aligned}
\Delta r_{n+1}^{-} & =r_{n+1}^{-}-r_{n}^{-} \\
& =(1-\beta)\left(r^{-}(\tilde{\overline{\boldsymbol{\sigma}}})-r_{n}^{-}\right)
\end{aligned}
$$

It is convenient to consider the effect of the irreversible strains through the definition of the effective stresses, since the Cauchy stresses are computed using the values of the effective stresses $\overline{\boldsymbol{\sigma}}^{ \pm}$and the two damage variables $d^{ \pm}$. This can be performed through the update of the effective stresses using equations (10) and (11) in a similar way to [33]

$\overline{\boldsymbol{\sigma}}_{n+1}=\lambda \tilde{\tilde{\boldsymbol{\sigma}}}$

\section{Tension and compression evolution laws consider re-} spectively the positive $G_{f}^{+}$and negative $G_{f}^{-}$fracture energies, as well as the characteristic finite element width $l_{\text {dis }}$ through the corresponding discrete softening parameter $H_{d}^{ \pm}$ensuring mesh-size independent energy dissipation according to the crack-band theory [43]. For the case of tension this is [44]

$H_{d}^{+}=\frac{l_{d i s}}{l_{m a t}^{+}-l_{d i s}}$

For the case of compressive damage, the discrete softening parameter $H_{d}^{-}$is defined as (see Appendix)

$H_{d}^{-}=\frac{1}{1-\beta}\left(\frac{l_{d i s}}{l_{m a t}^{-}-l_{d i s}}\right)$

The above definition is consistent with the crack band-width approach yielding objective results for different values of $\beta$ by considering the contributions to the dissipated energy of the evolution of the irreversible strains and the compressive damage. In the above, the 
material characteristic length $l_{m a t}^{ \pm}$for tension and compression is

$l_{m a t}^{ \pm}=\frac{2 E G_{f}^{ \pm}}{\left(f^{ \pm}\right)^{2}}$

The use of the discrete softening parameter of equation (20) results in an objective representation of the dissipated energy $G f^{-}$. This is illustrated in Figure 2 for the case of a cyclic uniaxial compressive loading using the proposed $H_{d}^{-}$of equation (20) instead of the one adopted in [45], which is the negative counterpart of equation (19). The use of the new softening modulus (Figure 2a) results in a consistent energy dissipation according to the input value of $G f^{-}$independently of the choice of $\beta$.

The effect of $\beta$ can now be visualised using equations (11) and (15) for the two limit cases of $\beta \rightarrow 0 ; 1$

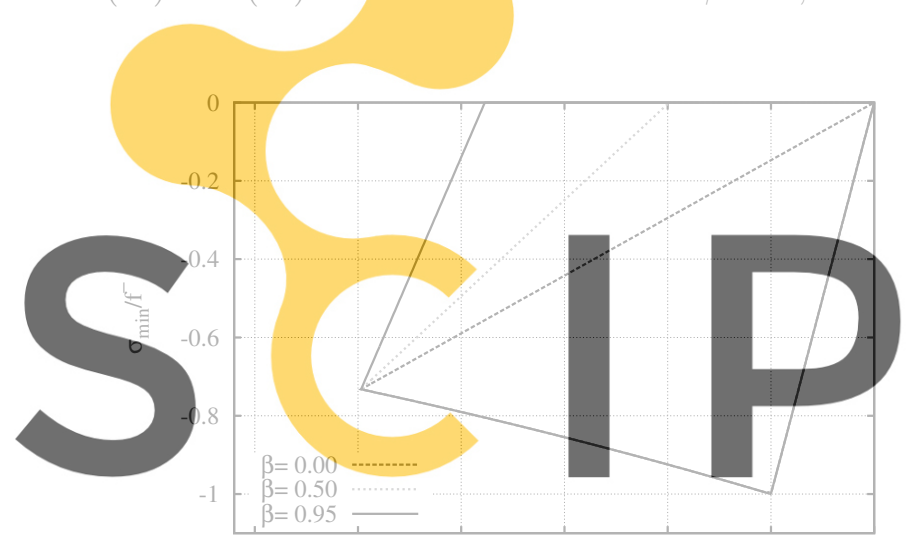

Register for 6 free-ăt https//wảww.šcipedia.com to

(a)

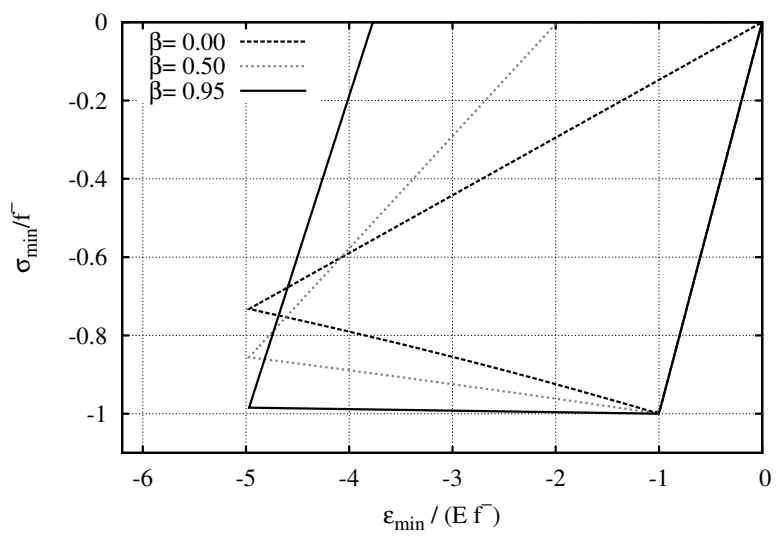

(b)

Fig. 2: Effect of the choice of the discrete softening parameter for compression to the softening behaviour for different values of $\beta$ : $H_{d}^{-}$(a) using equation (20), (b) using the formulation in [45].

$$
\begin{aligned}
\beta=0 & \Rightarrow \Delta r^{-}=r^{-}(\tilde{\tilde{\boldsymbol{\sigma}}})-r_{n}^{-} \\
& \Rightarrow \Delta \boldsymbol{\epsilon}_{n+1}^{i}=0 \\
\beta \rightarrow 1 & \Rightarrow \Delta r^{-}=0 \\
& \Rightarrow \Delta d_{n+1}^{-}=0
\end{aligned}
$$

The first case, with $\beta=0$, recovers the standard continuum damage mechanics model without any evolution of irreversible strains. Contrariwise, for $\beta \rightarrow 1$ the effective stresses are updated such that there is no evolution of the equivalent stress $\tau^{-}$and correspondingly $r^{-}$. For this case, there is no compressive damage evolution and the total incremental strains at this step will be irreversible. A value of $\beta$ within 0 and 1 , gives a proportional evolution of both irreversible strains and compressive damage. The above effect is illustrated in Figure 2a.

\subsection{Irreversible strains under shear loading}

As implied by their definition in equation (11), irre-

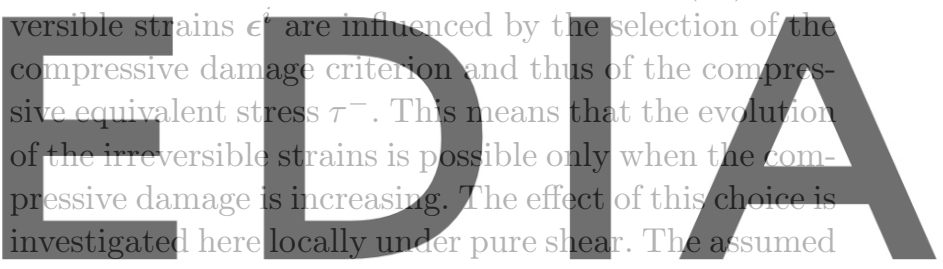

material parameters for this demonstrative example are

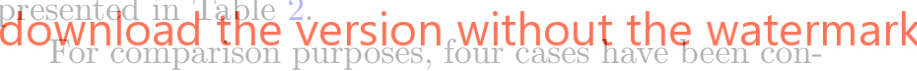
sidered with different definitions of $\tau^{-}$. In the first one, referred hereafter as Case-1, the $\tau^{-}$is calculated using only the negative effective stress tensor $\left(\bar{J}_{2}{ }^{-}, \bar{I}_{1}{ }^{-}\right.$, $\left.\sigma_{\max }^{-}, \sigma_{\min }^{-}\right)$as

$$
\tau^{-}=H\left[-\bar{\sigma}_{\min }^{-}\right] \frac{1}{1-a}\left[\sqrt{3 \bar{J}_{2}^{-}}+a{\overline{I_{1}}}^{-}+b\left\langle\bar{\sigma}_{\max }^{-}\right\rangle\right]
$$

The above definition using the negative part of the effective stress tensor, results in an "open" $d^{-}$damage surface in the shear quadrants, as shown in Figure 1 for the plane-stress case. This means that in this local test, compressive damage under pure shear is triggered only when the minimum principal stress reaches the uniaxial compressive strength of the material. For the other three cases, the equivalent stress of Eq. (5) is used that is a function of the total effective stress tensor $(\overline{\boldsymbol{\sigma}})$ with $\kappa_{1}=0.0 ; 0.5 ; 1.0$ for Case- 2 , Case- 3 and Case- 4 , respectively. The shapes of the four damage surfaces are presented qualitatively in Figure 1. Each case has 


\begin{tabular}{lllllll}
\hline$f^{+}$ & $f^{-}$ & $G_{f}^{+}$ & $G_{f}^{-}$ & $E$ & $v$ & $\beta$ \\
{$[M P a]$} & {$[M P a]$} & {$\left[J / m^{2}\right]$} & {$\left[J / m^{2}\right]$} & {$[M P a]$} & {$[-]$} & {$[-]$} \\
\hline \hline 0.2 & 5.0 & 50.0 & 10000 & 3500 & 0.2 & 0.50 \\
\hline
\end{tabular}

Table 2: Material parameters used in the elemental shear tests.

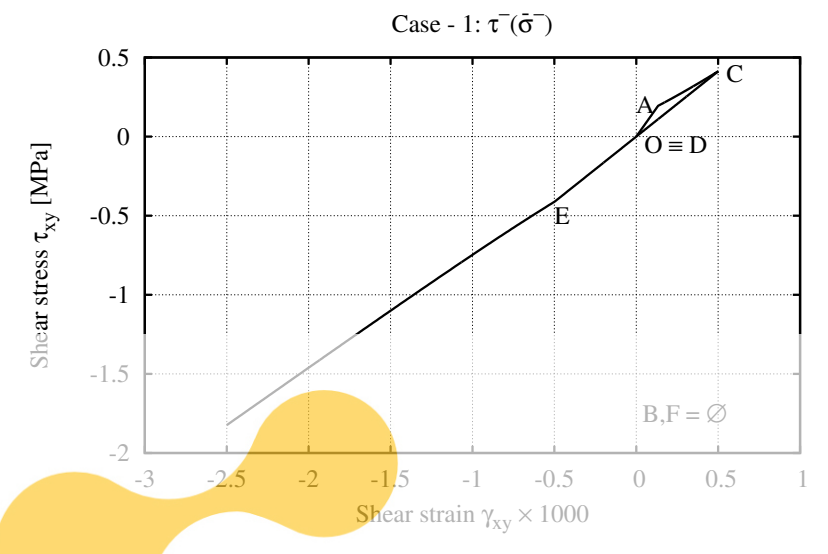

(a)

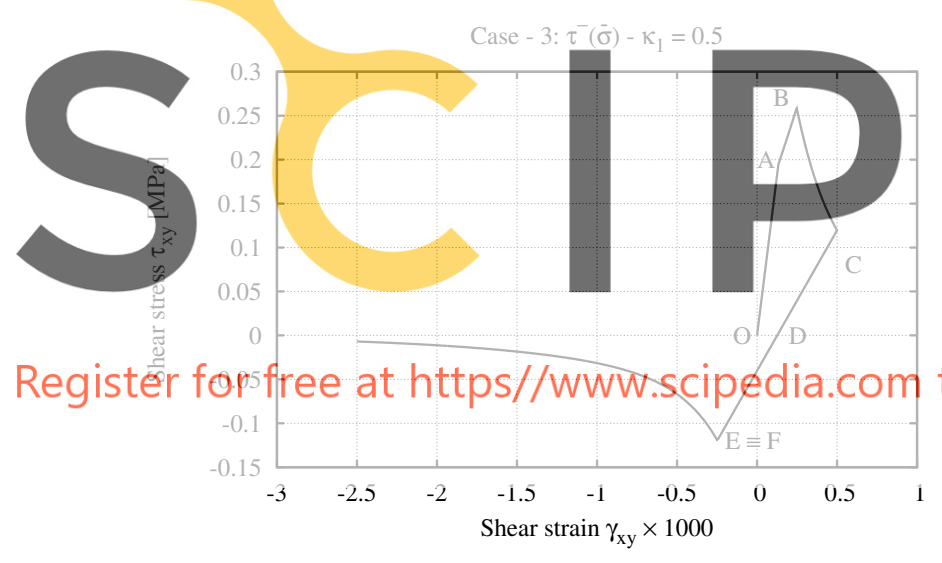

(c)

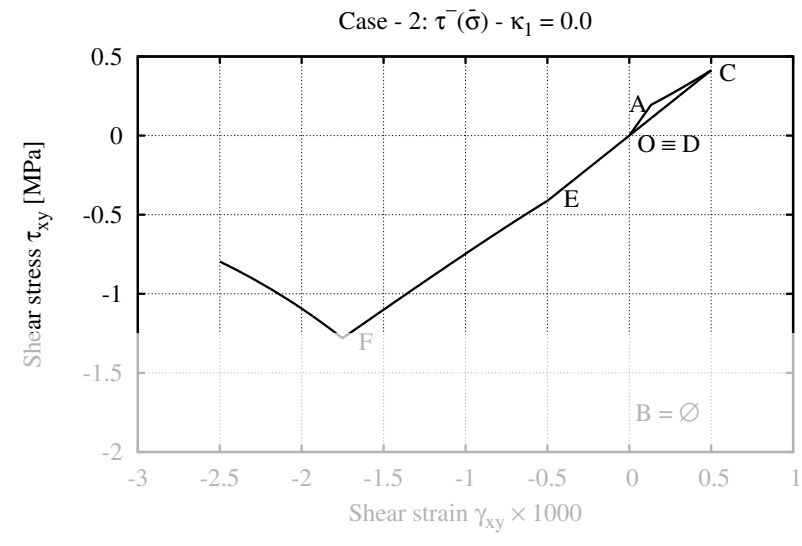

(b)

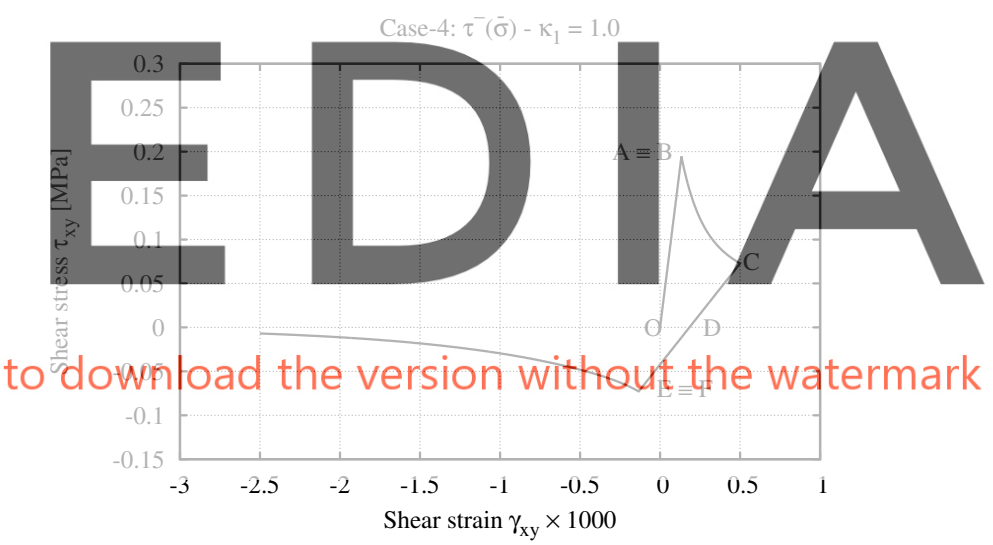

(d)

Fig. 3: Response under shear reverse loading for different assumptions about the compressive damage criterion. (a) Case $-1\left(\tau^{-}=\tau^{-}\left(\overline{\boldsymbol{\sigma}}^{-}\right)\right)$, (b) Case $-2\left(\tau^{-}=\tau^{-}(\overline{\boldsymbol{\sigma}})\right.$ and $\left.\kappa_{1}=0.0\right)$, (c) Case $-3\left(\tau^{-}=\tau^{-}(\overline{\boldsymbol{\sigma}})\right.$ and $\left.\kappa_{1}=0.5\right),(\mathrm{d})$ Case $-4\left(\tau^{-}=\tau^{-}(\overline{\boldsymbol{\sigma}})\right.$ and $\left.\kappa_{1}=1.0\right)$. Point A: Triggering of tensile damage, Point B: Triggering of the compressive damage, Point C: Maximum shear deformation for the first stage of the loading, Point D: Irreversible deformation for $\tau_{x y}=0$, Point E: Activation of the tensile damage, Point F: Activation of the compressive damage.

been subjected to a shear deformation composed of two stages: (a) loading up to a shear strain of $\gamma_{x y}=0.5 \times$ $10^{-3}$ and unloading to zero, (b) loading up to a shear strain of $\gamma_{x y}=-2.5 \times 10^{-3}$.

Figure 3 presents the response under shear for the four different cases. The letters appearing in the graphs refer to the following conditions occurring during the two loading stages: Point "A" triggering of tensile dam- age, Point "B" triggering of compressive damage, Point " $C$ ' maximum shear deformation for the first stage of loading, Point "D" Irreversible deformation for $\tau_{x y}=0$, Point "E" activation of the tensile damage during the second stage of loading, Point "F" activation of the compressive damage during the second stage of loading.

The part O-A in all graphs is the same and it represents the linear behaviour until reaching the $d^{+}$damage 
surface. After this point, the response in the four models depends on the specific assumption of the negative equivalent stress $\tau^{-}$, i.e. to the specific shape of the $d^{-}$ failure surface in the the second and fourth quadrants of Figure 1. For Case-1 and Case-2, the compressive failure criterion $\Phi^{-}$is not reached for the given maximum shear strain of $\gamma_{x y}=0.5 \times 10^{-3}$. This results in the increase of the shear capacity during the loading path A-C (see Figures 3a-b). An additional consequence of this is that there is no evolution of irreversible strains since the $d^{-}$failure surface is not reached (paths C$\mathrm{D}$ in Figures 3a-b). On the contrary, evolution of irreversible strains occurs for both Case- 3 and Case-4, since there is evolution of the compressive damage $d^{-}$ within the loading path A-C (Figures 3c-d). In Case-4, the overlapping of the tensile $d^{+}$and compressive $d^{-}$ failure surfaces results in the triggering of the tensile and compressive damage at the same time $(\mathrm{B} \equiv \mathrm{C})$ and in the earlier evolution of the irreversible strains. For this reason, the unloading path ( $\mathrm{C}-\mathrm{D}$, Figures $3 \mathrm{c}-\mathrm{d}$ ) is less sharp than the one in Case-3, resulting in larger irreversible strains.

The damage occurring during the first stage of a

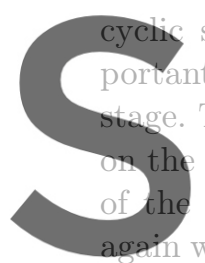
static loading analysis $(\mathrm{O}-$
nt effect during the reverse
The stiffness during the rev
$d^{+}$and $d^{-}$values attained
cyclic loading analysis. D
when the effective stresses $\tau^{-}$
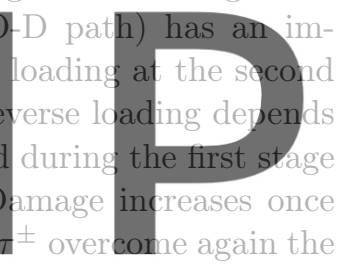

attained thresholds $r^{ \pm}$(Point E for $d^{+}$and Point F for

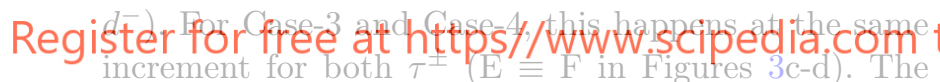
capacity of Case 2 keeps increasing up to the satisfaction of the failure criterion $\Phi^{-}$(Point F, Figure $3 \mathrm{~b}$ ). However, this does not happen for Case-1, resulting in an increasing shear capacity.

The above analyses show that the description of the unilateral effect under shear cyclic loading, and thus the definition of the unloading stiffness, requires the careful calibration of the equivalent stresses $\tau^{ \pm}$as well as the magnitude of the irreversible strains through the $\beta$ parameter.

\section{A tracking algorithm for intersecting and multi-directional cracks}

The simulation of localized cracks within the macromodelling approach is achieved through the use of a local crack-tracking algorithm. The aim of the algorithm is to identify the elements crossed by propagating cracks at each time/load increment. The crack is then simulated through the use of a nonlinear stress-strain rela- tionship for the elements within the crack path, while the ones outside maintain a linear elastic response.

Tracking algorithms are used in different numerical approaches developed for the simulation of localized cracks in quasi-brittle materials (see [46]). Despite the diversity of the applied fields, their function is always the same, i.e. the identification and localization of strains in a limited part of the structure, where cracks are expected to propagate. The methodology used here is based on the algorithm presented in [20, 21, 22, 32]. The local nature of this crack-tracking technique enables the identification of the crack trajectories from information already available at each step of a finite element analysis, i.e. the stress state of each finite element. In this way, computational cost, in terms of time and memory resources, is kept to values comparable to the classical smeared crack approach (see [32]). This strategy aims to a good balance between accuracy and efficiency, which is of primary importance in problems involving multiple and intersecting cracks, as is the case of cyclic loads in masonry structures.

This section presents the novel developments making possible the simulation of intersecting and multi-

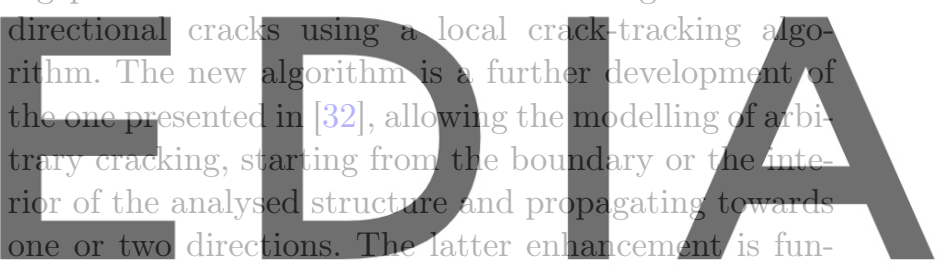

damental to simulate the response of structures expe-

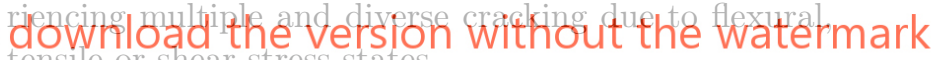 \\ The following of this section focuses on the details} of the novel algorithm. Firstly, a general introduction on the tasks of the tracking algorithm is presented. This is followed by the up to date assumptions regarding the modelling of crack initiation under monotonic loading. Modelling of crack propagation and intersection is presented in section 3.3. Finally, the section closes with the novel approach to simulate multi-directional cracking.

\subsection{Structure of the tracking algorithm}

The crack-tracking algorithm presented here is called at the beginning of each numerical increment before the calculation of the stress-state and has two tasks: (a) to identify the origin of new cracks and (b) to recognize the crack path and the finite elements pertaining to it. The output of the crack-tracking subroutine is a flag system used to distinguish elements within a crackpath from those outside. In this way, the two groups of elements follow different constitutive behaviour. Here, the continuum damage model presented in Section 2 is 


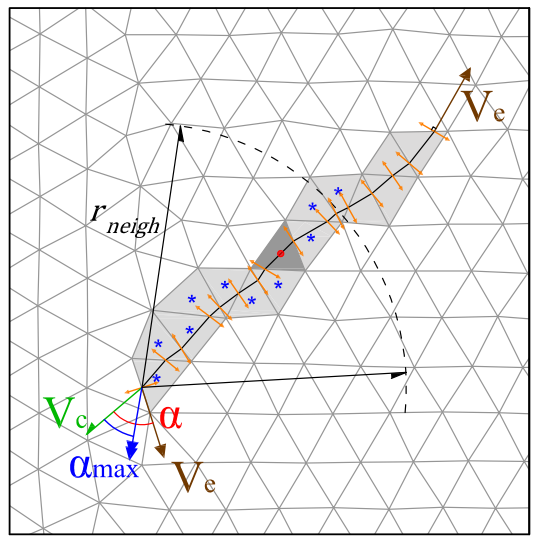

(a)

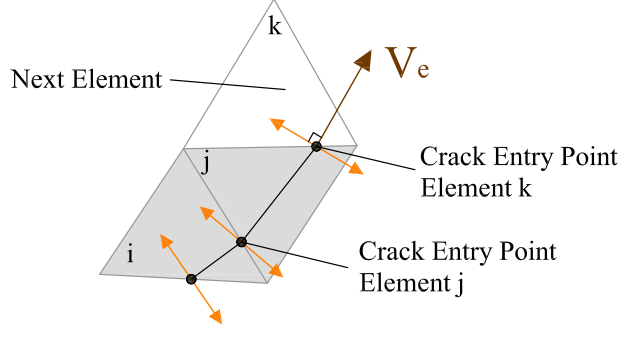

(b)

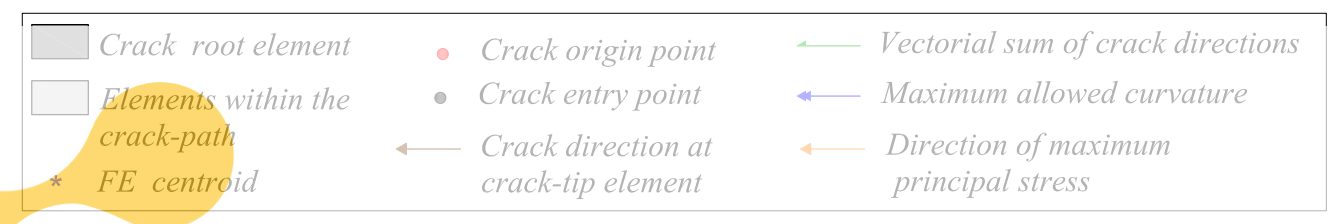

Fig. 4: Illustration of the crack propagation and use of the maximum curvature criterion for a new internal crack.
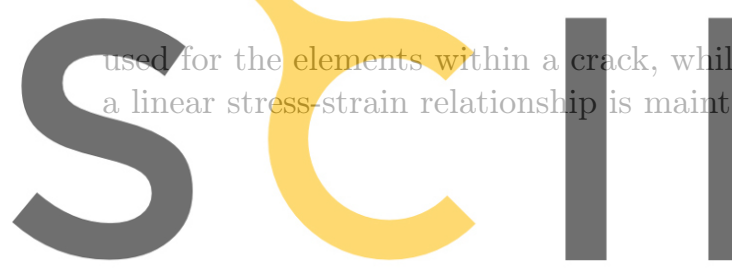

3.2 Crack initiation I - Monotonic Loading

\section{Register for free at https//www.scipedia.com to}

New cracks originate from elements that satisfy the failure condition as defined by Eq. (8). No restrictions are posed to the location of the crack origins in the used mesh as presented in [32]. For cracks initiating at corner or at internal elements, the centroid of the triangular element is selected as the crack origin. For the case of a crack starting at an element with one side on the boundary, the crack origin is located at the midpoint of the boundary edge. A minimum distance is defined between existing and new cracks, referred hereafter as exclusion radius $r_{\text {excl }}$ [22]. If two finite elements satisfy the failure criterion at the same time/load increment and the distance between them is less than the exclusion radius, the one with the highest value of the tensile equivalent stress $\tau^{+}$(see Eq. (4)) is selected as a crack origin. The value of the exclusion radius can be defined according to the structural problem or the fabric of the material analysed as shown in [22, 23, 32]. Apart from this functionality, the definition of an exclusion radius averts the initiation of secondary spurious cracking surrounding the crack-tip [27, 47], a pathology that may spoil the correct damage localization.

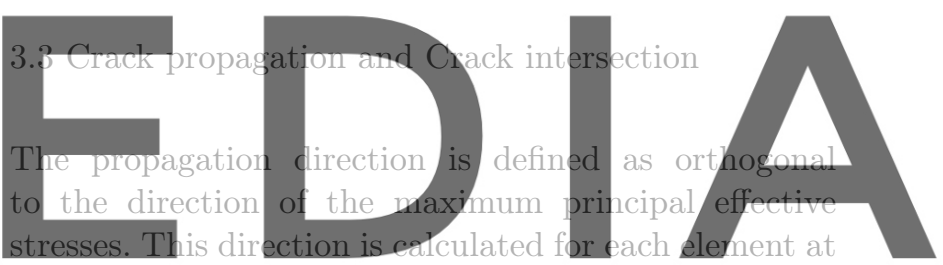

the crack front (crack tip element) using a stress tensor

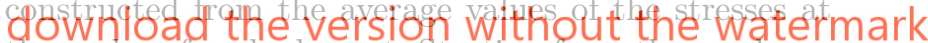
the nodes of each element. Starting from the crack entry point, and using a vector with the aforementioned direction ( $V_{e}$ in Figure 4$)$, the next potential element of the crack is identified and the crack entry coordinates are saved. Figure 4a presents an example with the propagation of an internal crack, while in Figure 4b shows a detail of the crack propagation and the crack entry coordinates on the wake of a crack tip. If the crack origin is an element lying on the boundary of the structure, the crack propagates in a single direction. This is the case of the flexural cracks at the corner of openings or at the base of piers. On the contrary, shear cracks can initiate from the interior of a masonry wall or a spandrel. To allow this, when the tracking algorithm recognizes that the crack origin is an element at the interior of the structure, the propagation of the crack is monitored towards two opposite orientations, as shown in Figure 4a. In this way, both shear and flexural cracks can be adequately modelled, as in the masonry frame wall presented in Figure 5.

In structures experiencing multiple cracking, it is unavoidable that cracks will meet other cracks during 


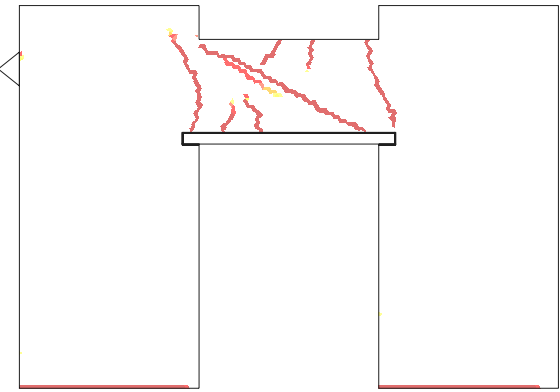

(a)

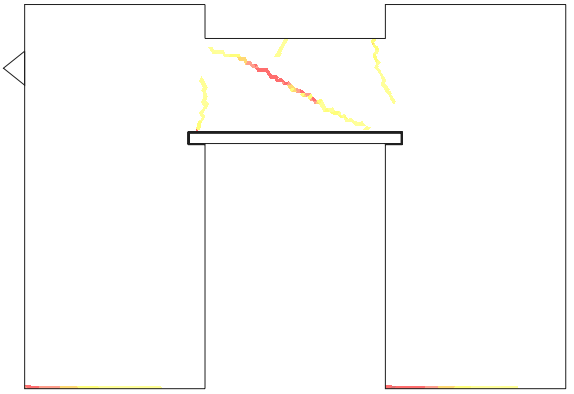

(b)

Fig. 5: Example of flexural and shear localized cracks in a masonry frame simulated under in-plane horizontal loading using the crack-tracking algorithm in [32]: (a) Tensile damage contour, and (b) Maximum principal strains at the end of the analysis.

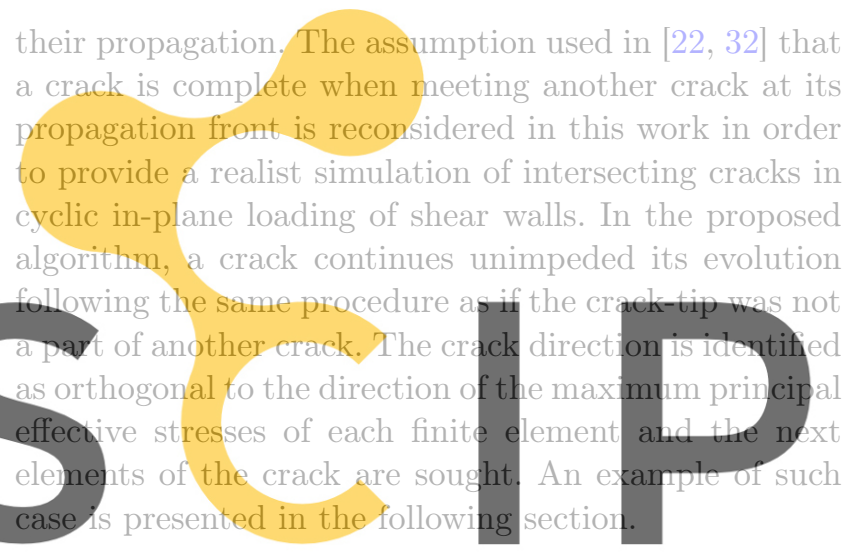

Register for free at https//www.scipedia.com to

In order to avoid sudden changes in the crack direction due to the poor estimation of the stresses arising from the use of standard finite elements, a correction of the crack propagation can be opportunely applied through the use of a maximum curvature criterion $[20,21,22]$. This criterion compares the crack propagation direction of the crack-tip element $V_{e}$ with the average crack direction $V_{c}$ (Figure $4 \mathrm{a}$ ). The latter is obtained as the average sum of the propagation directions of the elements in the crack path lying within a distance lower than $r_{\text {neigh }}$ from the crack-tip. If the propagation direction diverges with an angle $\alpha$ larger than $\alpha_{\text {max }}$, then the next element of the crack is sought using the average crack direction $V_{c}$ instead of $V_{e}$. This correction is illustrated in Figure 4. The value of the $r_{\text {neigh }}$ defines the length of the crack behind the crack tip that will be considered in the computation of the vector $V_{c}$ (see Figure $4 \mathrm{a}$ ). The maximum curvature angle $\alpha_{\max }$ determines the margins of the allowed crack rotation. The effect of these numerical parameters, as well as the criteria for their determination, are detailed in $[22,32]$.

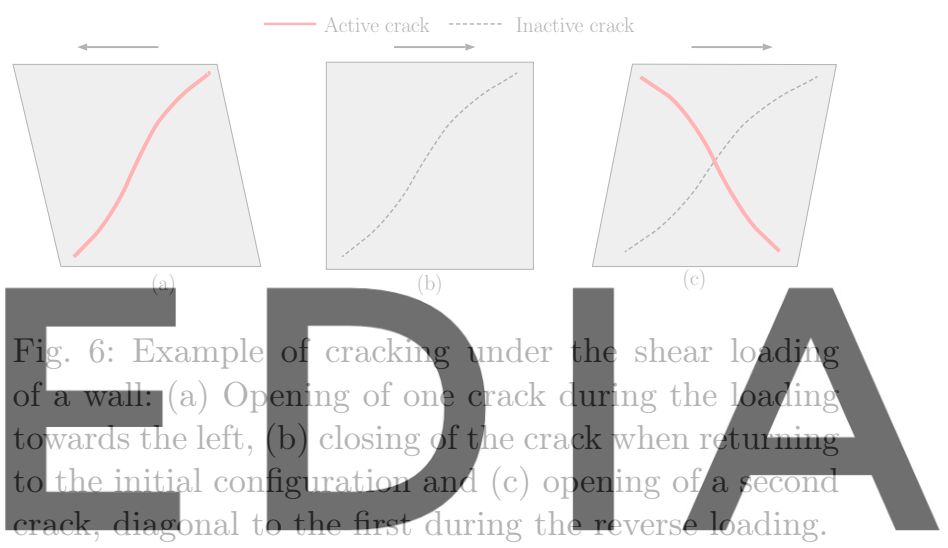

\section{download the version without the watermark 3.4 Crack initiation II - Multi-directional cracks}

The criteria for crack initiation, presented in section 3.2 , define that new cracks can start from a certain distance from existing ones, which is determined by the exclusion radius $r_{\text {excl }}$. This procedure is robust for structural cases with monotonic loading, where the crack directions do not show sudden changes during the loading history (see previous works [22, 23, 31, 32]). However, cyclic loading, such in earthquakes or wind loads, may induce multi-directional cracking to the structure, referring to cracks initiating in the same region of existing cracks but with a different direction. To illustrate this, consider the simplified case of a shear wall (Figure 6) subjected to cyclic shear loading at its top (restraining the rotation). The top displacement towards the left produces a shear crack, starting from the middle of the wall and propagating towards its two ends (Figure $6 \mathrm{a}$ ). The reversal of the loading cycle finds the wall at its initial configuration with the crack closed (Figure $6 \mathrm{~b}$ ). The loading in the opposite direction results in the opening of a new crack that propagates diagonally 


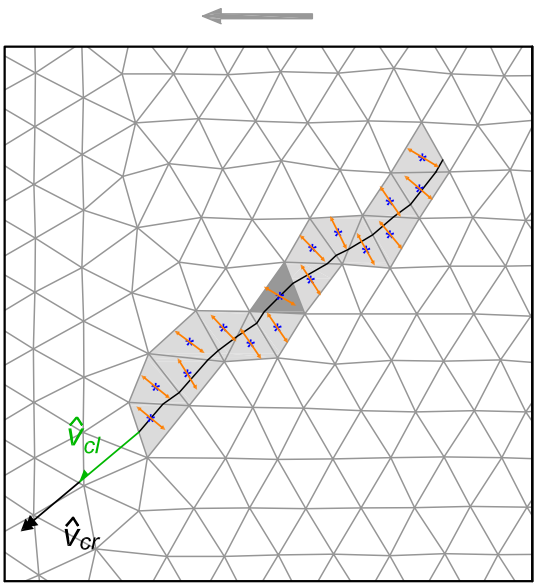

(a)

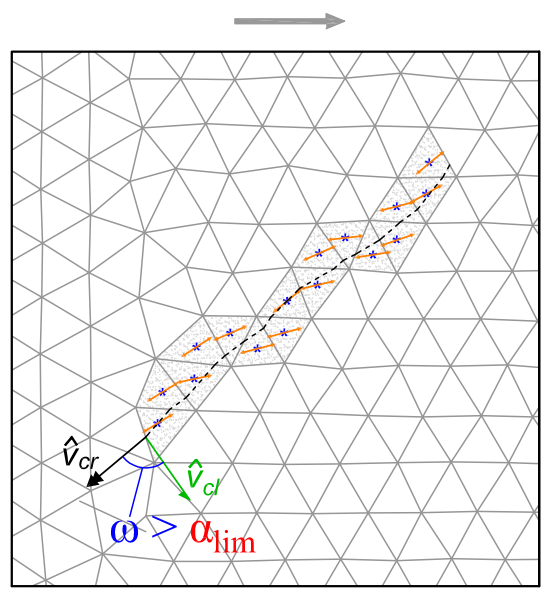

(b)

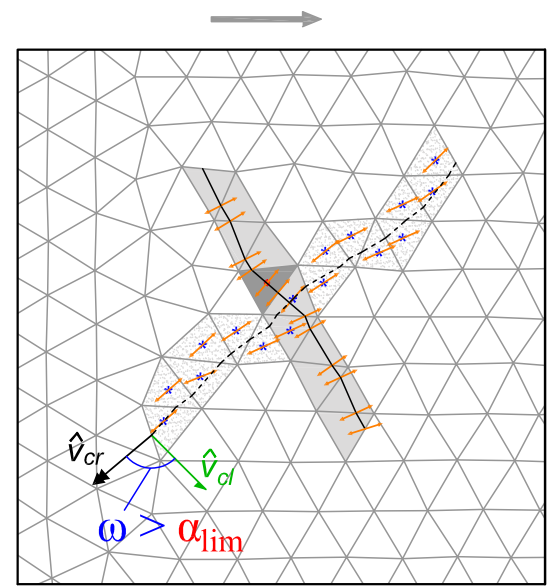

(c)

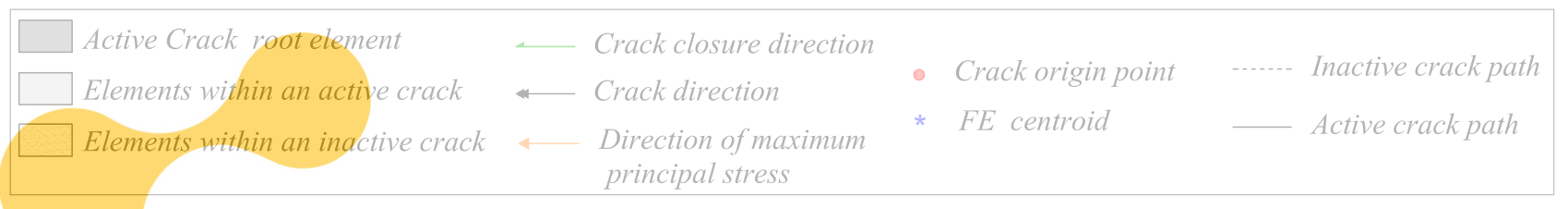

Fig. 7: Crack propagation in the middle of a shear wall: (a) propagation of a shear crack for the loading towards

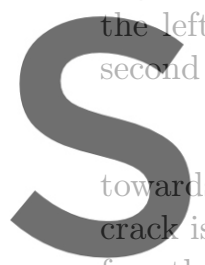
(b) deactivation of the crack next to the inactive
s the other two corners
is completely closed, the sti
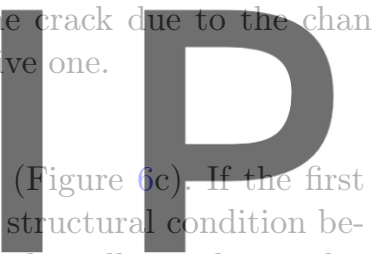

fore the opening of the two cracks will very be similar resulting to the possible origin of the second crack very Register for free iatohttps.stww.w.scipedia.com to

The accurate simulation of such cases needs a different approach regarding crack initiation from the one presented in Section 3.2. For the specific case of the shear wall in Figure 6, this approach must allow the initiation of the second crack at the finite element that satisfies the failure criterion after the reversal of the loading cycle, independently of its distance to the closed crack.

The modelling of cases involving the initiation of cracks due to different stress-states at a region of existing cracks is possible through the categorization of cracks into "active" and "inactive". Prior to the definition of these two categories, two new versors are introduced:

- Crack direction $\hat{v}_{c r}$ : a versor denoting the average direction of a consolidated crack. The crack direction of a crack is calculated using the crack entry coordinates at each element within the total length of the crack.

- Crack closure direction $\hat{v}_{c l}$ : a versor denoting the average direction orthogonal to the maximum

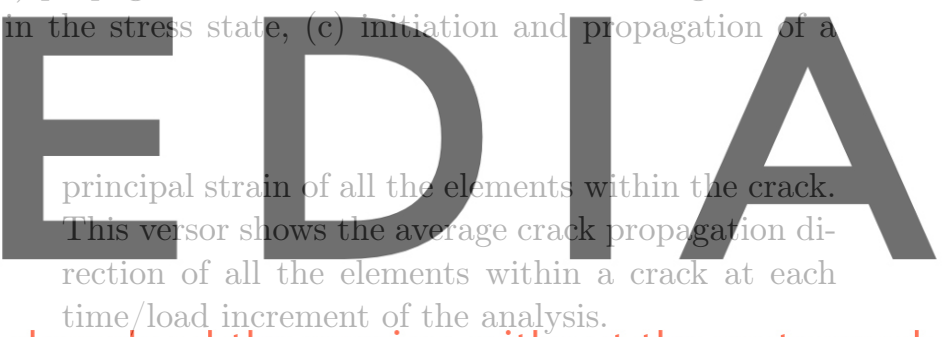

time load increment of the analysis.

download the version without the watermark

These two versors are updated at the end of each time/load increment for each of the consolidated cracks. A crack $i$ is defined as "active" if the angle between its crack direction versor $\hat{v}_{c r}^{i}$ and its crack closure direction versor $\hat{v}_{c l}^{i}$ is lower than a predefined limit angle $a_{l i m}$. The crack status is alternatively expressed through the use of the following function

$H^{i}\left[\left|\hat{v}_{c r}^{i} \cdot \hat{v}_{c l}^{i}\right|-\cos \left(a_{\text {lim }}\right)\right]=\left\{\begin{array}{ll}0 & \text { Inactive crack } \\ 1 & \text { Active crack }\end{array} \quad i=1, N_{c r}\right.$

where $(\cdot)$ is the dot product, the subscript $i=1, N_{c r}$ is used to denote the versor values for each crack of the total existing cracks $N_{c r}$ and $H[\bullet]$ is the Heaviside step function.

Following the above, an element will be the crack origin when all of the following criteria are satisfied:

(i) The failure condition, as defined by the constitutive model (Eq. 8), is reached. 
(ii) There is no "active" crack within a distance defined by the exclusion radius $r_{\text {excl }}$.

This means that new cracks can initiate at locations where the current stress-state has resulted in the closing of the pre-existing cracks. To illustrate this, Figure 7 illustrates how the algorithm models the two shear cracks at the middle of the wall for the example of Figure 6 . Figure 7 a shows the stress-state within the crack at the moment of the opening of the crack during the movement towards the left. The two versors of the crack $\left(\hat{v}_{c r}\right.$ and $\hat{v}_{c l}$, shown in one of the two crack-tips of the crack) coincide, as the crack direction has been defined according to the crack-propagation direction of each element within it. The crack is active and can continue its propagation. Figure $7 \mathrm{~b}$ presents the stress state for a step during the reversal of the cycle. The wall is now deforming towards the right and the existing crack is closed. This can be seen by the direction of the principal stresses which have rotated comparing to when the crack was open. The crack closure $\left(\hat{v}_{c r}\right)$ and crack direction versors $\left(\hat{v}_{c l}\right)$ form an angle which is greater than the limit angle $\alpha_{l i m}$. The existing crack is therefore identified as inactive, and new cracks are allowed to open. This happens at a later instance, shown in Figure $7 \mathrm{c}$, when a crack initiates very close to the existing crack and propagates thereafter.

The use of a limit angle as a way to identify the closure of existing cracks and the possibility of new ones to open resembles the concept used to model multiple cracks at constitutive level in the smeared crack models by de Borst and Nauta [48]. A similar concept was used also by Song \& Belytschko aiming to model crack branching for propagating cracks [47] under dynamic fracture. Likewise, the limit angle introduced in this work $\left(\alpha_{l i m}\right)$ is a numerical parameter aiming to deactivate the use of the exclusion radius when the strain state of the crack has exceeded the margins set by equation (27) and thus to allow new cracks to emerge close to existing ones.

\section{Numerical simulation of a shear wall under cyclic loading}

\subsection{Experimental and numerical set-up}

In this section, the proposed numerical model is calibrated through the comparison with an experimental case study. The test was performed by Antoine et al. [49] and considers the in-plane response of a masonry wall under quasi-static shear loading. The wall has a width of $1.00 \mathrm{~m}$ and a height of $1.35 \mathrm{~m}$, while its thickness is $0.25 \mathrm{~m}$. The masonry is constructed using an English bond pattern with unit dimensions of $55 \times 120 \times 250 \mathrm{~mm}^{3}$ and $10 \mathrm{~mm}$ mortar joint thickness. A vertical load of $150 \mathrm{kN}$ was distributed at the top part of the wall and was maintained constant during the whole experiment, aiming to represent gravitational loading acting on the top of the masonry wall during a seismic event. The seismic action was reproduced by applying an horizontal displacement at the top of the wall. Proper boundary conditions were applied to avoid its top and bottom rotation. Figure 8a shows a sketch of the experimental set-up adopted during the execution of the cyclical shear tests.

The experimentally obtained shear load against displacement graph is presented in Figure 8b. The wall presents the typical behaviour for shear dominating failure. This is characterised by an overall drop in the capacity with increasing load cycles and appreciable energy dissipation throughout the whole loading history, as represented by the area of the hysteretic cycles of the load-displacement curve. The quasi-brittle character of the shear failure is due to the decrease of strength produced by the formation of two systems of crossing diagonal cracks in the wall $[49,50]$.

In this work, the experiment is reproduced by using the continuum damage model and the crack-tracking algorithm presented in Sections 2 and 3. The wall has been discretized using 3 -noded constant-strain triangles (2836 nodes). The material parameters are presented in Table 3. The compressive strength, the density and the Young's modulus are equal to the values obtained through experimental tests on stack bond prisms reported in $[51,50]$. The rest of the mechanical properties of the materials, and the parameters of the constitutive model have been chosen in order to calibrate correctly the shear response of the material, on the basis on the discussion presented in Section 2.2. To this aim, a value of $\kappa_{1}=0.15$ has been chosen which is similar to the value used in other available studies on masonry shear walls [19, 39, 41]. Regarding the crack-tracking parameters, the exclusion radius has been set equal to $r_{\text {excl }}=0.20 \mathrm{~m}$, which is a value between the length and the width of the masonry units. The limit angle used for the activation-deactivation of the cracks under the cyclic loading is $\alpha_{l i m}=40^{\circ}$. This value means that three additional cracks with an orientation varying by $40^{\circ}$ can initiate next to an existing crack. To visualize this, and considering that the exclusion radius has been defined equivalent to the units size, the above value implies that a total of four cracks with different orientations can initiate from the same unit. For the investigated case of a shear wall under cyclic loading, this value seems reasonable as it can allow 


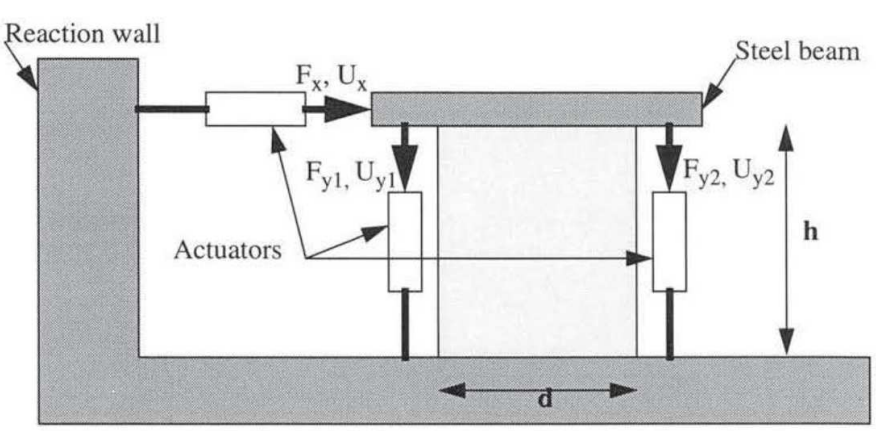

(a)

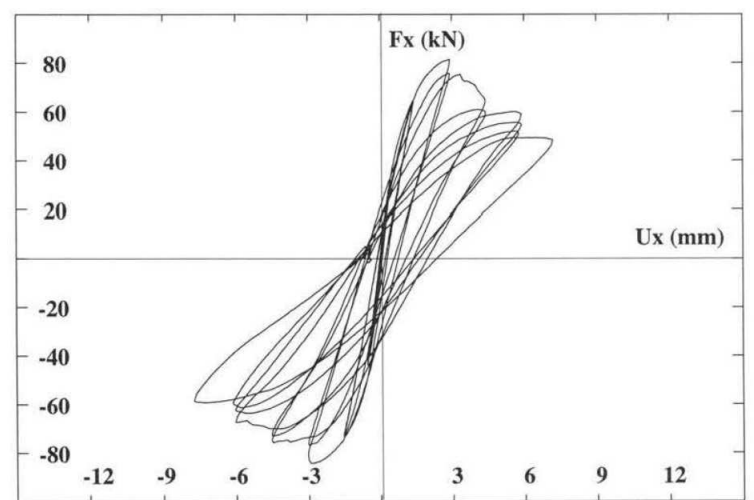

(b)

Fig. 8: (a) Experimental set-up and (b) experimentally obtained force against displacement graph of the tested masonry wall. Pictures adapted from [49].

\begin{tabular}{lllllllll}
\hline$f^{+}$ & $f^{-}$ & $G_{f}^{+}$ & $G_{f}^{-}$ & $E$ & $\rho$ & $v$ & $\beta$ & $\kappa_{1}$ \\
{$[M P a]$} & {$[M P a]$} & {$\left[J / m^{2}\right]$} & {$\left[J / m^{2}\right]$} & {$[M P a]$} & {$\left[k g / m^{3}\right]$} & {$[-]$} & {$[-]$} & {$[-]$} \\
\hline \hline 0.15 & 6.2 & 80.0 & 14000 & 1490 & 1750 & 0.2 & 0.95 & 0.15 \\
\hline
\end{tabular}

Table 3: Material parameters for the masonry shear wall.

the formation of diagonal cracking due to shear failure. The parameters for the maximum curvature criterion are $\alpha_{\max }=25^{\circ}, r_{\text {neigh }}=0.60 \mathrm{~m}$. These values of the parameters showed to be adequate to avoid sudden changes in the crack direction as reported in previous works of the authors [22].

The wall has been analysed under plane stress conditions and the simulation has been performed in two stages. The first one includes the application of the selfweight and the vertical compressive load at the top of the wall. In the second stage, a cyclic horizontal displacement is applied at the top of the wall with increasing magnitude, as in the experiment. Vertical displacement at the top is restrained, precluding the rotation of the wall. The numerical solution is performed in an incremental manner. At each time/load increment, the nonlinear equations are solved with the use of a modified Newton-Raphson (using the secant stiffness matrix). Convergence is achieved when the ratio between the norm of the iterative residual forces and the norm of the total external forces is lower than $10^{-2}$. Calculations are performed using an enhanced version of the finite element software COMET [52], while preand post-processing with $\mathrm{GiD}$ [53], both developed in CIMNE, Barcelona.

\subsection{Numerical results}

Figure 9 presents the contours of the tensile $d^{+}$and compressive $d^{-}$damage indices, as well as that of the maximum principal strains at the end of the analysis. The wall is dominated by diagonal shear cracking, which is in agreement with the reported experimental damage pattern $[49,50]$. There is evidence of flexural cracking at the lower and bottom parts of the wall, as well as at the two sides. Despite the opened cracks during the loading history, the main degradation of the wall is concentrated at the two diagonal cracks that have opened in the middle of the wall, as shown by the contour of the maximum principal strains. The use of the crack-tracking algorithm results in a very clear representation of the localized cracks, aiding the identification of the occurring collapse mechanism at the wall.

Figure 10a presents the damage evolution and Figure $10 \mathrm{~b}$ the state of the cracks for the opposite top displacements of this cycle. For demonstration purposes, the contour value 1 (red) is used for the active cracks and -1 (white) for the inactive ones, while the elements outside of the crack maintain value equal to 0 (orange) (Figure $(10 \mathrm{~b})$ ). It is visible that all cracks are active for the maximum displacement of the first cycle with a loading direction towards the right. Two flexural cracks at the two corners of the wall consist the first occurring 


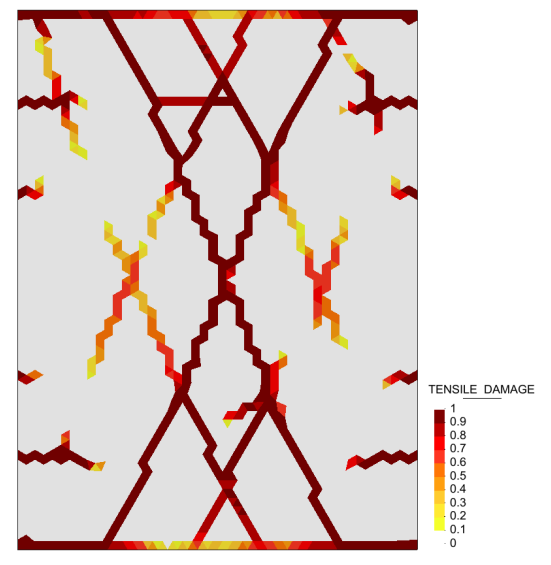

(a)

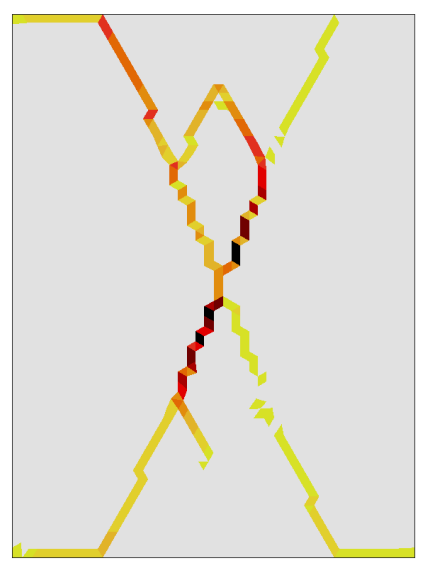

(b)

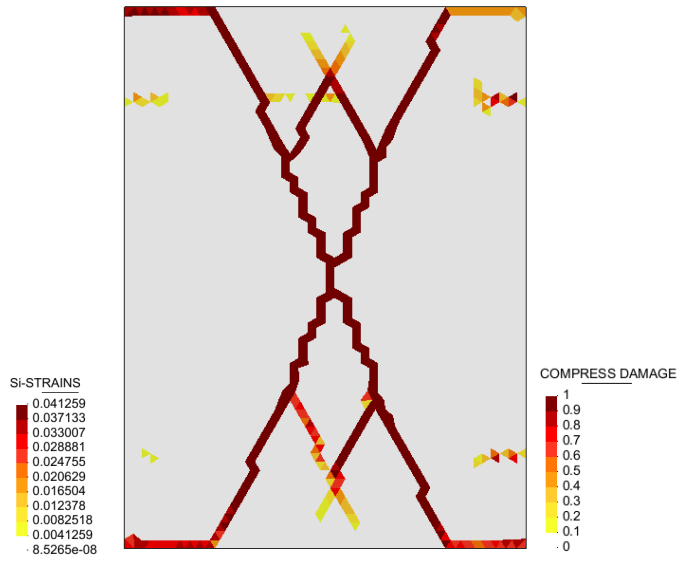

(c)

Fig. 9: Contours of: (a) the $d^{+}$damage index, (b) the maximum principal strains at the end of the analysis, (c) the $d^{-}$damage index at the end of the analysis.
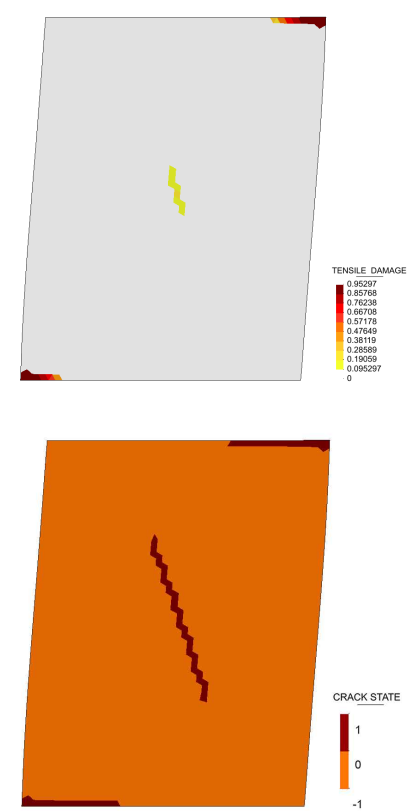
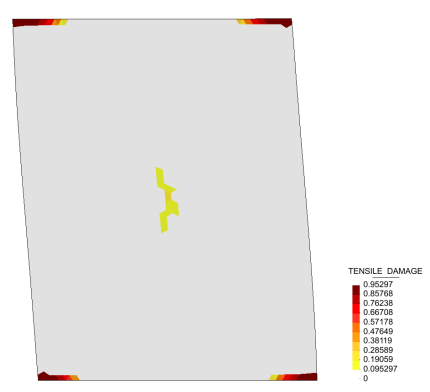

(a)

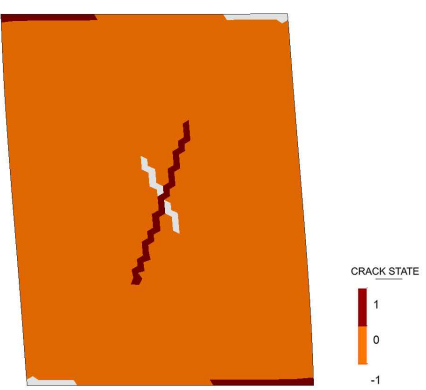

(b)

Fig. 10: (a) Tensile damage contour $d^{+}$and (b) crack state during the first loading cycle for the maximum displacement towards the right (left column) and the maximum displacement towards the left (right column) (deformed mesh $\times 100)$.

damage. After those, a shear crack initiated at the middle of the wall (left column of Figure 10). The results with the same top displacement but opposite direction are presented in the right column of Figure 10. All the previously opened cracks have now closed and are correctly identified as inactive. New horizontal cracks appear at the two opposite corners, as well as a diagonal shear crack at the middle of the wall, intersecting the existing crack. The proposed approach is capable to simulate both the correct initiation of new cracks close to the existing ones, as well as their intersection.

Figure 11 presents the damage pattern during the loading cycle with a maximum displacement at the top equal to $\delta= \pm 3.0 \mathrm{~mm}$. After the initial damage shown in Figure 10, additional shear cracks have opened, with the deformation, however, localizing at the initial diagonal crack at the middle of the wall (see center column of Figure 11). Some flexural cracks initiate at the two 

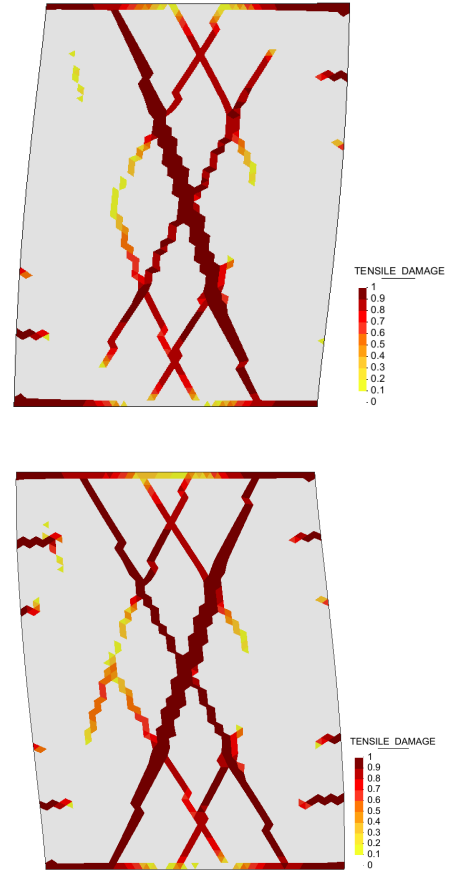

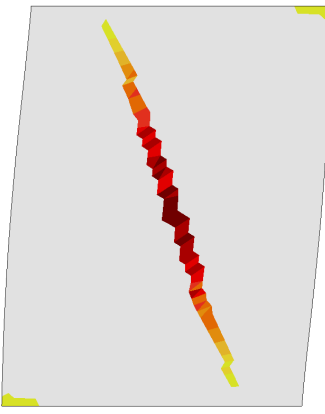

(a)

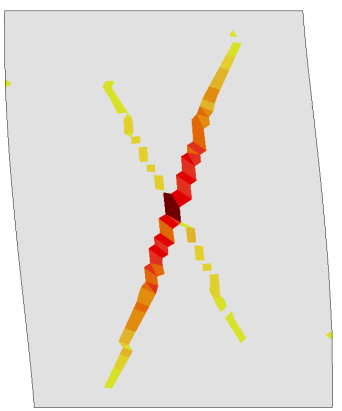

(b)
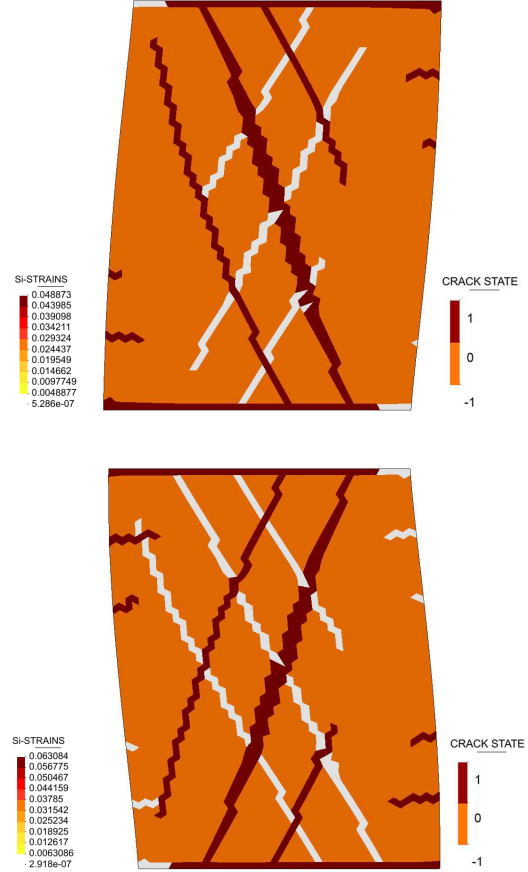

Fig. 11: Contours of tensile $d^{+}$damage (left), maximum principal strains (center) and crack state (right) for a top displacement of $3.0 \mathrm{~mm}$ towards the right (a) and left (b), (deformed mesh $\times 50)$.

lateral sides of the wall and propagate horizontally towards the interior. Note that the cracks opened during an opposite loading direction have been correctly identified as closed (right column in Figure 11).

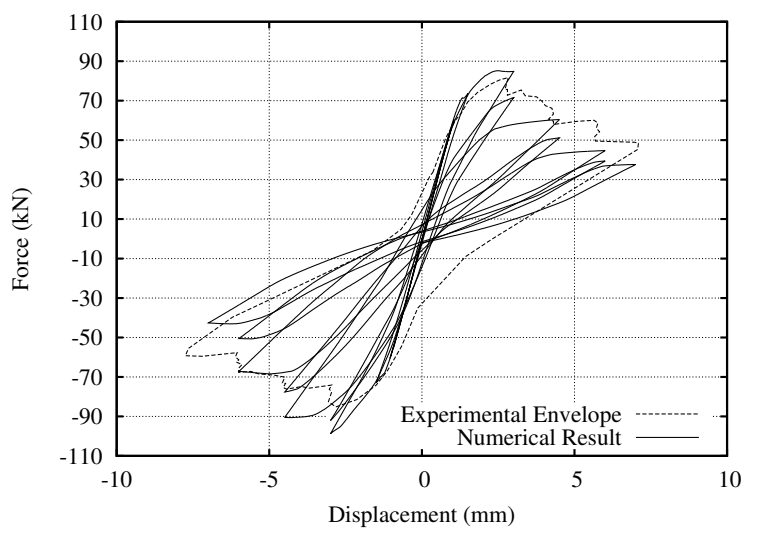

Fig. 12: Force against horizontal displacement graphs for the masonry shear wall obtained through the numerical analysis.

The response of the simulated wall in terms of horizontal load against horizontal displacement is presented in Figure 12. The numerical simulation captures the shear failure of the wall, which is characterized by an overall softening branch and stiffness degradation after exceeding the maximum capacity. The first cycles of the loading are characterized by an almost linear behaviour, due to the small amount of damage as shown in Figure 10. A significant drop in the capacity occurs after the complete degradation of the diagonal cracks at the middle of the wall, which occurs during the loading cycles with the maximum displacement of $30 \mathrm{~mm}$, as in the experiment. After that, the increase of the displacement results in the drop of the stiffness due to the degradation of the additional shear cracks, yielding the final damage pattern and strain state of Figure 9.

The used constitutive model is able to represent satisfactorily the cyclic damaging behaviour of the wall. The slight underestimation of the magnitude of the experimental envelope of the hysteretic loops is due to the specific nature of the used damage model, with the association of the evolution of the irreversible strains to only that of the compressive damage variable $d^{-}$. In fact, as can be seen in Figure 9c, compressive damage $d^{-}$appears only at the two diagonal cracks at the middle of the wall, making them the only source of the irreversible deformations. 

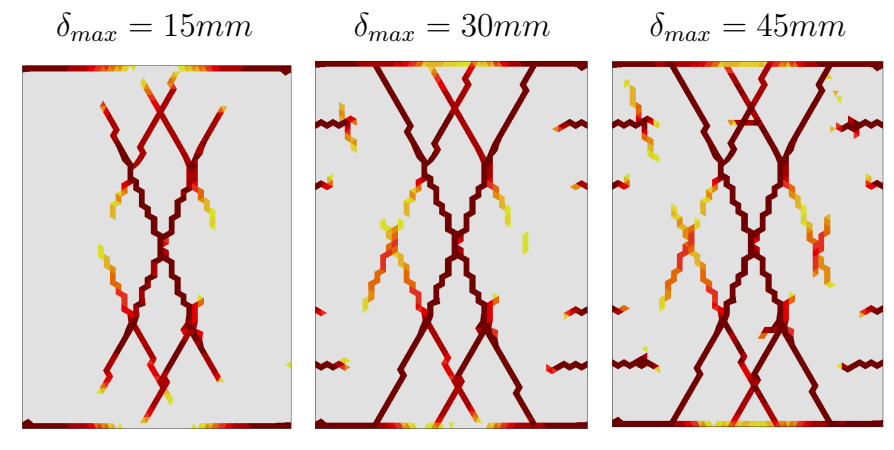

$\delta_{\max }=70 \mathrm{~mm}$

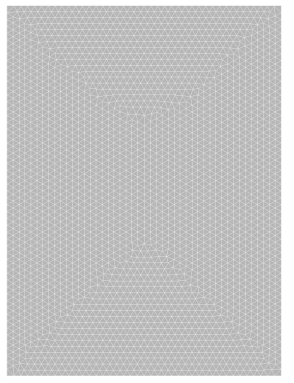

(a)
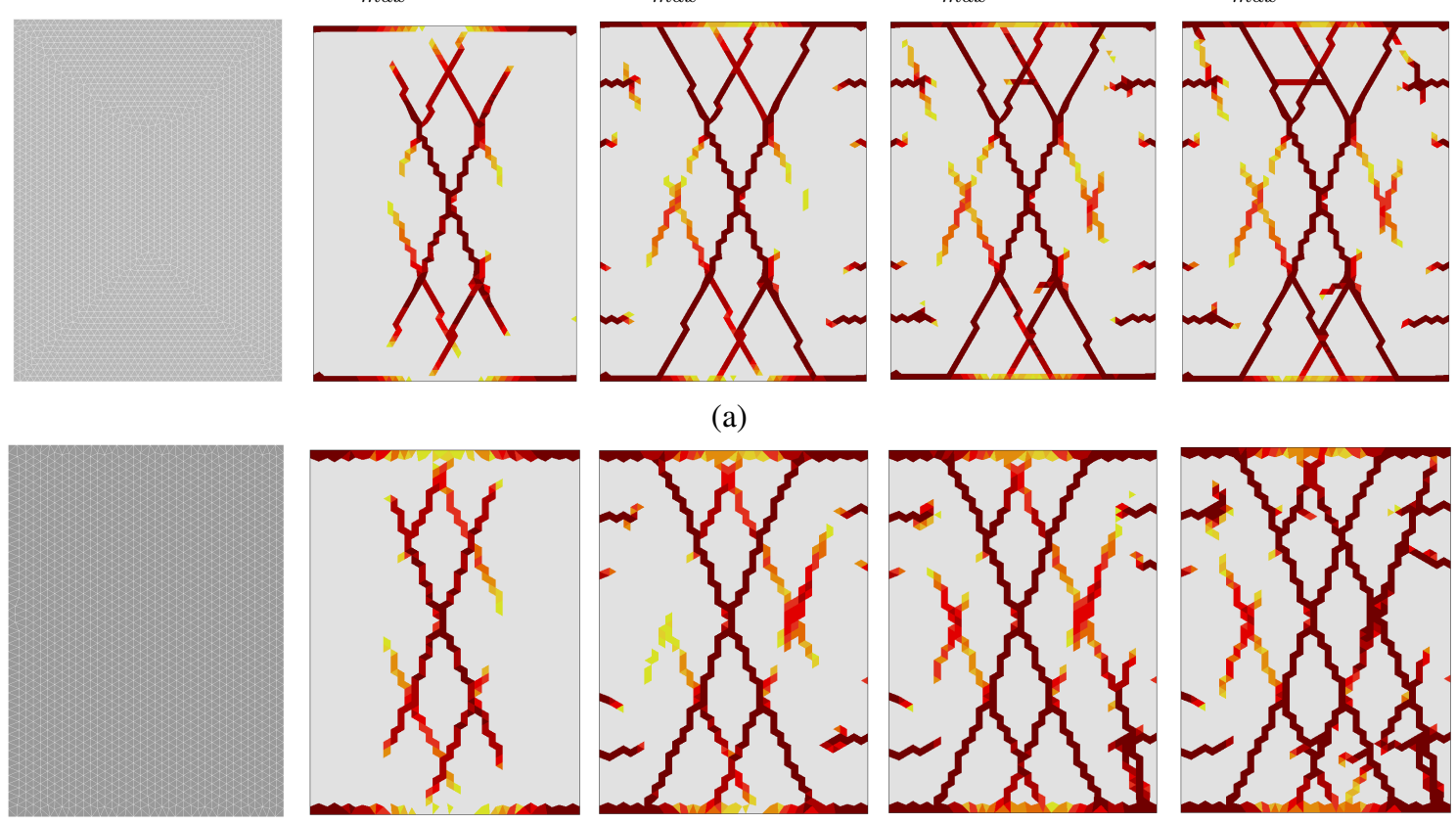

(b)

Fig. 13: The used discretization (first column) and the contours of the tensile damage at the end of the various cycles with increasing maximum displacement $\delta_{\max }$ : (a) Mesh-A, (b) Mesh-B

\subsection{Mesh-dependency study}

The tracking algorithm has shown to improve the mesh objectivity in structural analyses of small [22, 31, 32] and large scale structures [54] with monotonically propagating cracks. In this section, the shear wall is analysed with an additional mesh aiming to study the mesh dependency of the presented approach for the more demanding case of cyclic loading. The first column of Figure 13 shows the different discretization used in the mesh dependency analyses. The first mesh, denoted hereafter as Mesh-A, is mesh used for the aforementioned results with 5470 elements. The second mesh, Mesh-B, presents a constant structured pattern in the greatest part of the wall with equilateral triangles making angles of $30^{\circ}, 90^{\circ}$ and $150^{\circ}$ with the horizontal axis and has a lower number of elements (3704) compared to Mesh-A.

The different columns of Figure 13 present the damage pattern at the end of the different cycles during the loading history. Diagonal shear cracking dominates the main part of the wall in both cases, while flexural cracks appear at the boundaries. The damage pattern is very similar for the greatest loading history of the wall, with some differences occurring in the last cycles, during which the coarser mesh (Mesh-B) presents some additional shear cracking.

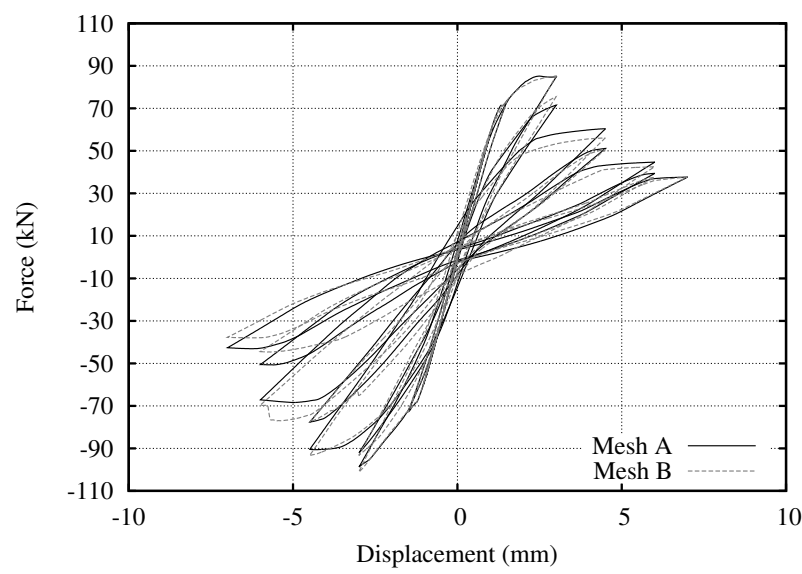

Fig. 14: Horizontal force against displacement at the top of the wall for Mesh-A and Mesh-B

Figure 14 illustrates the force against the applied displacement graphs for the two meshes overlapped. The two analyses bare important similarities. The predicted capacity of the wall shows a variation of less than $3 \%$ and is attained at the second loading cycle for a displacement of $30 \mathrm{~mm}$. Following that, the structure presents a softening response with dropping capacity and energy dissipation due to the further opening of the cracks. As presented in Figure 13, after the first two 
loading cycles most of the cracks have already formed, and the diagonal cracks at the middle of the wall as well as the boundary cracks at the top and bottom ends of the wall have completely opened. Upon this point, the numerical responses reflect the small differences regarding the positions of the cracks and the appearance of more cracks at the coarser mesh (Mesh-B), slightly alternating the residual response of the wall.

\section{Conclusions}

This paper has presented the enhancement of the macro-modelling finite element method with a cracktracking algorithm for the simulation of the behaviour of masonry structures under in-plane cyclic loading. A novel methodology has been proposed to simulate intersecting and multi-directional cracks that occur in masonry under cyclic actions with a local crack-tracking technique. This has been achieved by setting suitable crack opening/closure criteria in the numerical algorithm. Cracking is represented at a local level using a continuum damage model with distinct representation of tensile and compressive damage. The constitutive model has been formulated to include the description of the evolution of irreversible deformations in a simplified way.

The enhanced macro-model has been used to simulate an experimentally tested shear masonry wall under cyclic quasi-static loading. The tracking algorithm has shown to be capable to simulate shear and flexural cracks propagating in multiple directions and intersecting among them. The results show that the used constitutive model can represent correctly the gradual decrease of the structural capacity during the cyclic loading process. The proposed approach can therefore properly account for complex damage typologies when cracks appear and propagate under shear cyclic condition.

The paper includes a discussion at constitutive level regarding the evolution of the irreversible strains under shear loading. A simple and explicit algorithmic formulation, together with a proper definition of the compressive damage surface, allow to consider the effect of irreversible strains both under shear and compression stress states.

\section{Compliance with ethical standards}

Conflict of interest The authors declare that they have no conflict of interest.
Funding This research has received the financial support from the MINECO (Ministerio de Economia y Competitividad of the Spanish Government) and the ERDF (European Regional Development Fund) through the MULTIMAS project (Multiscale techniques for the experimental and numerical analysis of the reliability of masonry structures, ref. num. BIA2015-63882-P).

\section{References}

1. S. Huerta, "The Analysis of Masonry Architecture: A Historical Approach," Architectural Science Review, vol. 51, no. 4, pp. 297-328, 2008.

2. P. Roca, M. Cervera, G. Gariup, and L. Pelà, "Structural Analysis of Masonry Historical Constructions. Classical and Advanced Approaches," Archives of Computational Methods in Engineering, vol. 17, pp. 299-325, jul 2010.

3. D. Theodossopoulos and B. Sinha, "A review of analytical methods in the current design processes and assessment of performance of masonry structures," Construction and Building Materials, vol. 41, pp. 990-1001, 2013.

4. D. Addessi, S. Marfia, E. Sacco, and J. Toti, "Modeling Approaches for Masonry Structures," The Open Civil Engineering Journal, no. 2, pp. 288-300, 2014.

5. A. Orduña and P. B. Lourenço, "Cap Model for Limit Analysis and Strengthening of Masonry Structures," Journal of Structural Engineering, vol. 129, no. 10, pp. 1367-1375, 2003.

6. P. Block, T. Ciblac, and J. Ochsendorf, "Real-time limit analysis of vaulted masonry buildings," Computers 8 Structures, vol. 84, pp. 1841-1852, nov 2006.

7. M. Gilbert, C. Casapulla, and H. Ahmed, "Limit analysis of masonry block structures with nonassociative frictional joints using linear programming," Computers \& Structures, vol. 84, pp. 873887, may 2006.

8. G. Milani, P. Lourenço, and A. Tralli, "Homogenised limit analysis of masonry walls, Part I: Failure surfaces," Computers \& Structures, vol. 84, pp. 166-180, jan 2006.

9. P. Roca, C. Molins, and A. R. Marí, "Strength Capacity of Masonry Wall Structures by the Equivalent Frame Method," Journal of Structural Engineering, vol. 131, no. 10, pp. 1601-1610, 2005.

10. S. Lagomarsino, A. Penna, A. Galasco, and S. Cattari, "TREMURI program: An equivalent frame model for the nonlinear seismic analysis of ma- 
sonry buildings," Engineering Structures, vol. 56, pp. 1787-1799, 2013.

11. R. Siano, V. Sepe, G. Camata, E. Spacone, P. Roca, and L. Pelà, "Analysis of the performance in the linear field of Equivalent-Frame Models for Regular and Irregular Masonry Walls," Engineering Structures, vol. In Press, 2017.

12. Y. Endo, L. Pelà, P. Roca, F. Porto, and C. Modena, "Comparison of seismic analysis methods applied to a historical church struck by 2009 L' Aquila earthquake," Bulletin of Earthquake Engineering, 2015.

13. S. Saloustros, L. Pelà, P. Roca, and J. Portal, "Numerical analysis of structural damage in the church of the Poblet monastery," Engineering Failure Analysis, vol. 48, pp. 41-61, 2014.

14. L. Pelà, M. Cervera, and P. Roca, "An orthotropic damage model for the analysis of masonry structures," Construction and Building Materials, vol. 41, pp. 957-967, 2013.

15. P. B. Lourenço and J. G. Rots, "Multisurface Interface Model for Analysis of Masonry Structures," Journal of Engineering Mechanics, vol. 123, no. 7, pp. 660-668, 1997.

16. L. Macorini and B. A. Izzuddin, "A non-linear interface element for 3D mesoscale analysis of brickmasonry structures," International Journal for $\mathrm{Nu}$ merical Methods in Engineering, vol. 85, pp. 15841608, mar 2011.

17. T. J. Massart, R. H. J. Peerlings, and M. G. D. Geers, "An enhanced multi-scale approach for masonry wall computations with localization of damage," International Journal for Numerical Methods in Engineering, vol. 69, pp. 1022-1059, jan 2007.

18. D. Addessi and E. Sacco, "A multi-scale enriched model for the analysis of masonry panels," International Journal of Solids and Structures, vol. 49, no. 6, pp. 865-880, 2012.

19. M. Petracca, L. Pelà, R. Rossi, S. Oller, G. Camata, and E. Spacone, "Multiscale computational first order homogenization of thick shells for the analysis of out-of-plane loaded masonry walls," Computer Methods in Applied Mechanics and Engineering, vol. 315, pp. 273-301, 2017.

20. R. Clemente, Análysis estructural de edificios históricos mediante modelos localizados de fisuración. Ph.d. thesis, Universitat Politècnica de Catalunya, 2006.

21. L. Pelà, Continuum Damage Model for Nonlinear Analysis of Masonry Structures. Ph.d. thesis, Universitat Politècnica de Catalunya, 2009.

22. M. Cervera, L. Pelà, R. Clemente, and P. Roca, "A crack-tracking technique for localized damage in quasi-brittle materials," Engineering Fracture Mechanics, vol. 77, no. 13, pp. 2431-2450, 2010.

23. P. Roca, M. Cervera, L. Pelà, R. Clemente, and M. Chiumenti, "Continuum FE models for the analysis of Mallorca Cathedral," Engineering Structures, vol. 46, pp. 653-670, 2013.

24. L. Pelà, M. Cervera, S. Oller, and M. Chiumenti, "A localized mapped damage model for orthotropic materials," Engineering Fracture Mechanics, vol. 124-125, pp. 196-216, 2014.

25. M. Cervera and M. Chiumenti, "Smeared crack approach: back to the original track," International Journal for Numerical and Analytical Methods in Geomechanics, vol. 30, no. 12, pp. 1173-1199, 2006.

26. A. Slobbe, M. Hendriks, and J. Rots, "Smoothing the propagation of smeared cracks," Engineering Fracture Mechanics, vol. 132, pp. 147-168, dec 2014.

27. J. Oliver and A. E. Huespe, "Continuum approach to material failure in strong discontinuity settings," Computer Methods in Applied Mechanics and Engineering, vol. 193, no. 30-32, pp. 3195-3220, 2004.

28. G. Meschke and P. Dumstorff, "Energy-based modeling of cohesive and cohesionless cracks via XFEM," Computer Methods in Applied Mechanics and Engineering, vol. 196, no. 21-24, pp. 2338-2357, 2007.

29. D. Dias-Da-Costa, J. Alfaiate, L. J. Sluys, and E. Júlio, "A comparative study on the modelling of discontinuous fracture by means of enriched nodal and element techniques and interface elements," International Journal of Fracture, vol. 161, no. 1, pp. 97-119, 2010.

30. Y. Zhang, R. Lackner, M. Zeiml, and H. a. Mang, "Strong discontinuity embedded approach with standard SOS formulation: Element formulation, energy-based crack-tracking strategy, and validations," Computer Methods in Applied Mechanics and Engineering, vol. 287, pp. 335-366, 2015.

31. S. Saloustros, L. Pelà, and M. Cervera, "A crack-tracking technique for localized cohesivefrictional damage," Engineering Fracture Mechanics, vol. 150, pp. 96-114, 2015.

32. S. Saloustros, L. Pelà, M. Cervera, and P. Roca, "Finite element modelling of internal and multiple localized cracks," Computational Mechanics, vol. 59, no. 2, pp. 299-316, 2016.

33. R. Faria and J. Oliver, A Rate Dependent PlasticDamage Constitutive Model for Large Scale Computations in Concrete Structures. CIMNE, 1993.

34. M. Cervera, J. Oliver, and R. Faria, "Seismic evaluation of concrete dams via continuum damage models," Earthquake Engineering \& Structural Dynam- 
ics, vol. 24, no. 9, pp. 1225-1245, 1995.

35. R. Faria, J. Oliver, and M. Cervera, "Modeling material failure in concrete structures under cyclic actions," Journal of Structural Engineering, no. December, pp. 1997-2005, 2004.

36. P. Roca, M. Cervera, L. Pelà, R. Clemente, and M. Chiumenti, "Viscoelasticity and Damage Model for Creep Behavior of Historical Masonry Structures," pp. 188-198, 2012.

37. L. Pelà, J. Bourgeois, P. Roca, M. Cervera, and M. Chiumenti, "Analysis of the Effect of Provisional Ties on the Construction and Current Deformation of Mallorca Cathedral," International Journal of Architectural Heritage, vol. in press, no. doi: 10.1080/15583058.2014.996920, 2014.

38. R. Faria, J. Oliver, and M. Cervera, "A strain-based plastic viscous-damage model for massive concrete structures," International Journal of Solids and Structures, vol. 35, pp. 1533-1558, may 1998.

39. M. Petracca, L. Pelà, R. Rossi, S. Oller, G. Camata, and E. Spacone, "Regularization of first order computational homogenization for multiscale analysis of masonry structures," Computational Mechanics, vol. 57, pp. 257-276, dec 2016.

40. J. Lubliner, J. Oliver, S. Oller, and E. Oñate, "A plastic-damage model for concrete," International Journal of Solids and Structures, vol. 25, no. 3, pp. 299-326, 1989.

41. M. Petracca, L. Pelà, R. Rossi, S. Zaghi, G. Camata, and E. Spacone, "Micro-scale continuous and discrete numerical models for nonlinear analysis of masonry shear walls," Construction and Building Materials, vol. In Press, 2017.

42. J. Oliver, M. Cervera, S. Oller, and J. Lubliner, "Isotropic damage models and smeared crack analysis of concrete," Proc. SCI-C Computer Aided Analysis and Design of Concrete Structures, no. February, pp. 945-957, 1990.

43. Z. Bazant and B. Oh, "Crack band theory for fracture of concrete," Materials and Structures, vol. 16, pp. 155-177, 1983.

44. M. Cervera and M. Chiumenti, "Mesh objective tensile cracking via a local continuum damage model and a crack tracking technique," Computer Methods in Applied Mechanics and Engineering, vol. 196, no. 1-3, pp. 304-320, 2006.

45. M. Cervera, "Viscoelasticity and Rate-dependent Continuum Damage Models, Monography N-79," tech. rep., Barcelona, 2003.

46. T. Rabczuk, S. Bordas, and G. Zi, "On threedimensional modelling of crack growth using partition of unity methods," Computers and Structures, vol. 88, no. 23-24, pp. 1391-1411, 2010.
47. J.-H. Song and T. Belytschko, "Cracking node method for dynamic fracture with finite elements," International Journal for Numerical Methods in Engineering, vol. 77, pp. 360-385, jan 2009.

48. R. de Borst and P. Nauta, "Non-orthogonal cracks in a smeared finite element model," Engineering Computations, vol. 2, no. 1, pp. 35-46, 1985.

49. A. Anthoine, G. Magenes, and G. Magonette, "Shear compression tensting and analysis of brick masonry walls," in 10th European Conference on Earthquake Engineering, (Vienna), pp. 1657-1662, 1994.

50. G. Magenes and G. M. Calvi, "In-plane seismic response of brick masonry walls," 1997.

51. L. Binda, G. Mirabella Roberti, C. Tiraboschi, and S. Abbaneo, "Measuring Masonry Material Properties," U.S.-Italy Workshop on Guidelines for Seismic Evaluation and Rehabilitation of Unreinforced Masonry Buildings, pp. 326-347, 1994.

52. COMET, "Coupled Mechanical and Thermal analysis, http://www.cimne.com/comet/," 2016.

53. GiD, "The personal pre and post-processor, http://www.gidhome.com/," 2016.

54. S. Saloustros, L. Pelà, M. Cervera, and P. Roca, "An enhanced finite element macro-model for the realistic simulation of localized cracks in masonry structures: A large-scale application," International Journal of Architectural Heritage, vol. In press, no. doi: 10.1080/15583058.2017.1323245, 2017.

\section{Appendix}

The rate of the mechanical dissipation $\gamma$ of the continuum damage model presented in Section 2 is [33]

$\dot{\gamma}=-\frac{\partial \psi}{\partial \epsilon^{i}}: \dot{\boldsymbol{\epsilon}}^{i}-\frac{\partial \psi}{\partial d^{+}} \dot{d}^{+}-\frac{\partial \psi}{\partial d^{-}} \dot{d}^{-}=\dot{\gamma}^{i}+\dot{\gamma}^{d} \geq 0$

where $\psi$ is the Helmholtz free energy and $\psi_{0}^{ \pm}$elastic free energies which have the following form [33]

$$
\begin{aligned}
& \psi=\left(1-d^{+}\right) \psi_{0}^{+}+\left(1-d^{-}\right) \psi_{0}^{-} \\
& \psi_{0}^{+}=\frac{1}{2} \overline{\boldsymbol{\sigma}}^{+}: \boldsymbol{C}_{\mathbf{0}}^{-1}: \overline{\boldsymbol{\sigma}} \\
& \psi_{0}^{-}=\frac{1}{2} \overline{\boldsymbol{\sigma}}^{-}: \boldsymbol{C}_{\mathbf{0}}^{-1}: \overline{\boldsymbol{\sigma}}
\end{aligned}
$$

It is visible from equation (28) that the evolutions of both damage $\dot{\gamma}^{d}$ and irreversible strains $\dot{\gamma}^{i}$ contribute to the total dissipation energy of the solid, with each part being 
$\dot{\gamma}^{i}=-\frac{\partial \psi}{\partial \boldsymbol{\epsilon}^{i}}: \dot{\boldsymbol{\epsilon}}^{i} \geq 0$

$\dot{\gamma}^{d}=-\frac{\partial \psi}{\partial d^{+}} \dot{d}^{+}-\frac{\partial \psi}{\partial d^{-}} \dot{d}^{-} \geq 0$

The total dissipated energy per unit volume $g_{f}$ is obtained as

$g_{f}=\int_{0}^{t} \dot{\gamma}^{i} d t+\int_{0}^{t} \dot{\gamma}^{d} d t$

The discrete softening parameter can be defined similarly to [44] considering an ideal uniaxial 1D compressive experiment, with a monotonic increment of the compressive strain (denoted hereafter as $\epsilon^{e}$ ) from an initial unstressed state to full degradation. During the loading, and considering equations (5) and (9) the stress threshold will be

$r^{-}=E \epsilon^{e}$

In such case the dissipation due to the damage evolution is (see [44])

$$
\begin{aligned}
\gamma^{d} & =\int_{0}^{t} \dot{\gamma}^{d} d t \\
& =\frac{1}{2 E} \int_{r 0}^{t} r^{2} d^{\prime} d r \\
& =\left(1+\frac{1}{H_{d}^{-}}\right) \frac{\left(f^{-}\right)^{2}}{2 E}
\end{aligned}
$$

The contribution of the irreversible strains to the total dissipated energy can be computed considering

$$
\begin{aligned}
\dot{\epsilon}^{i} & =\beta \frac{E \dot{\epsilon}}{E \epsilon^{e}} \epsilon^{e} \\
& =\beta \dot{\epsilon} \\
& =\frac{\beta}{(1-\beta)} \dot{\epsilon}^{e}
\end{aligned}
$$

and

$-\frac{\partial \psi}{\partial \epsilon^{i}}=\left(1-d^{-}\right) r^{-}$

Using the above equations (39c) and (40), the dissipated energy due to the evolution of the irreversible strains is

$$
\begin{aligned}
\gamma^{i} & =\int_{r 0}^{t}\left(1-d^{-}\right) r^{-} \frac{\beta}{(1-\beta)} \dot{\epsilon}^{e} d t \\
& =\int_{r 0}^{r}\left(1-d^{-}\right) r^{-} \frac{\beta}{(1-\beta)} \frac{1}{E} d r \\
& =\frac{\beta}{1-\beta} \frac{\left(f^{-}\right)^{2}}{E} \frac{1}{2 H_{d}^{-}}
\end{aligned}
$$

By virtue of equations (36) as well as (41) and considering that the total dissipation should be equal to $G f^{-}$, the updated softening modulus is derived as

$H_{d}^{-}=\frac{1}{1-\beta}\left(\frac{l_{d i s}}{l_{m a t}^{-}-l_{d i s}}\right)$

Note that for the limit case of $\beta=1$, equation (39b) limits to $\dot{\epsilon}^{i}=\dot{\epsilon}$ and consequently $\dot{\epsilon}^{e}=0$. This results in $\Delta r=0$, which means that there is no damage evolution and hence no softening. In that case, energy is dissipated only due to the evolution of the irreversible strains, which using equations (32), (35) and (40) will be

$$
\begin{aligned}
\gamma^{i} & =E \epsilon_{0}^{e} \int_{0}^{t} \dot{\epsilon} d t \\
& =E \epsilon_{0}^{e} \int_{\epsilon_{0}}^{e} d \epsilon \\
& =E \epsilon_{0}^{e}\left[\epsilon-\epsilon_{0}^{e}\right]
\end{aligned}
$$

with $\epsilon_{0}^{e}$ being the elastic strain at the peak strength. According to the above, the dissipation will keep increasing linearly with the increase of the strains, resembling the behaviour of a perfectly plastic material. 